\title{
Adaptive evolution of multiple-variable exons and structural diversity of drug-metabolizing enzymes Can $\mathrm{Li}$ and Qiang Wu*
}

\author{
Address: Department of Human Genetics, University of Utah, Salt Lake City, Utah 84112, USA \\ Email: Can Li - canli@genetics.utah.edu; Qiang Wu* - qwu@genetics.utah.edu \\ * Corresponding author
}

Published: 2 May 2007

BMC Evolutionary Biology 2007, 7:69 doi:10.1/86/147|-2/48-7-69

This article is available from: http://www.biomedcentral.com/I47I-2/48/7/69

(C) $2007 \mathrm{Li}$ and $\mathrm{Wu}$; licensee BioMed Central Ltd.

This is an Open Access article distributed under the terms of the Creative Commons Attribution License (http://creativecommons.org/licenses/by/2.0), which permits unrestricted use, distribution, and reproduction in any medium, provided the original work is properly cited.
Received: 26 February 2007

Accepted: 2 May 2007

\begin{abstract}
Background: The human genome contains a large number of gene clusters with multiple-variablefirst exons, including the drug-metabolizing UDP glucuronosyltransferase (UGTI) and I-branching $\beta-I, 6-\mathrm{N}$-acetylglucosaminyltransferase (GCNT2, also known as IGNT) clusters, organized in a tandem array, similar to that of the protocadherin $(P C D H)$, immunoglobulin $(I G)$, and T-cell receptor (TCR) clusters. To gain insight into the evolutionary processes that may have shaped their diversity, we performed comprehensive comparative analyses for vertebrate multiple-variable-firstexon clusters.
\end{abstract}

Results: We found that there are species-specific variable-exon duplications and mutations in the vertebrate Ugtl, Gcnt2, and Ugt2a clusters and that their variable and constant genomic organizations are conserved and vertebrate-specific. In addition, analyzing the complete repertoires of closely-related Ugt2 clusters in humans, mice, and rats revealed extensive lineage-specific duplications. In contrast to the Pcdh gene clusters, gene conversion does not play a predominant role in the evolution of the vertebrate Ugtl, Gent2 and Ugt2 gene clusters. Thus, their tremendous diversity is achieved through "birth-and-death" evolution. Comparative analyses and homologous modeling demonstrated that vertebrate UGT proteins have similar three-dimensional structures each with $\mathrm{N}$-terminal and $\mathrm{C}$-terminal Rossmann-fold domains binding acceptor and donor substrates, respectively. Molecular docking experiments identified key residues in donor and acceptor recognition and provided insight into the catalytic mechanism of UGT glucuronidation, suggesting the human UGTIAI residue histidine $39(\mathrm{H} 39)$ as a general base and the residue aspartic acid I5I (DI5I) as an important electron-transfer helper. In addition, we identified four hypervariable regions in the $\mathrm{N}$-terminal Rossmann domain that form an acceptor-binding pocket. Finally, analyzing patterns of nonsynonymous and synonymous nucleotide substitutions identified codon sites that are subject to positive Darwinian selection at the molecular level. These diversified residues likely play an important role in recognition of myriad xenobiotics and endobiotics.

Conclusion: Our results suggest that enormous diversity of vertebrate multiple variable first exons is achieved through birth-and-death evolution and that adaptive evolution of specific codon sites enhances vertebrate UGT diversity for defense against environmental agents. Our results also have interesting implications regarding the staggering molecular diversity required for chemical detoxification and drug clearance. 


\section{Background}

Alternative splicing is one of the most important mechanisms to generate molecular diversity in vertebrates. A large number of alternatively spliced genes that have multiple"variable" first exons have been identified in the human genome, including protocadherin $(P C D H)$, UDPglucuronosyltransferase (UGT), plectin (PLEC1), neuronal nitric oxide synthase (NOS1), and glucocorticoid receptor (GR) genes [1]. In particular, the closely-linked vertebrate Pcdh $\alpha$ and $\gamma$ clusters have a striking genomic organization each containing more than a dozen variable first exons and three downstream "constant" exons [2-7]. Alternative splicing of each variable exon to the common set of constant exons generates diverse functional mRNA molecules that encode a large number of cadherin-like cell-surface proteins in the central nervous system (CNS). Comparative analyses suggest that gene duplication, gene conversion, and variable exon mutation play important roles in vertebrate Pcdh evolution [3-5]. In addition, adaptive selection of specific residues in the ectodomains enhances mammalian Pcdh diversity [5]. Combinatorial interactions between these Pcdh proteins contribute to the establishment and maintenance of trillions of diverse yet very specific neuronal connections in the vertebrate CNS.

In the vertebrate adaptive immune system, the immunoglobulin $(I g)$, T-cell receptor $(T c r)$, and major histocompatibility complex (Mhc) gene clusters provide the enormous diversity required for immune defense. The Ig and $\mathrm{Tcr}$ clusters are organized into variable and constant regions. Gene duplications and somatic DNA rearrangements generate tremendous diversity for mammalian Ig and Tcr molecules. Moreover, positive natural selection operates on the complementarity-determining regions (CDRs) of the IG and TCR proteins to increase their diversity [8-11]. The Mhc genes are also clustered. The encoded MHC proteins (both class I and II human HLA molecules) have a deep peptide-binding groove formed by a $\beta$-sheet bottom floor and two $\alpha$-helix side walls $[12,13]$. Each MHC protein can bind a large set of different peptides. In addition, most of the polymorphic residues on the $\beta$-sheet floor and two $\alpha$-helix side walls point towards the peptide-binding groove and serve as ligands for numerous processed antigens [14-16]. Finally, diversity-enhancing overdominant selection operates on the antigen-binding sites of both class I and II MHC proteins enabling them to recognize diverse processed antigens $[11,17,18]$. Vertebrate animals evolved these three gene families through birth-and-death evolution of repeated duplication and mutation, in conjunction with positive selection, to remove a staggering number of different foreign antigens in a highly specific fashion $[11,19]$.
Vertebrate animals also remove hundreds of thousands xeno- and endobiotic lipophilic compounds from their bodies by converting them to water-soluble glucuronides through glucuronidation [20]. This detoxification pathway converts lipophilic aglycones to hydrophilic molecules and facilitates their excretion from the body. Glucuronidation is catalyzed by members of the UGT glucuronosyltransferase proteins in the endoplasmic reticulum (ER) [21,22]. Vertebrate UGT proteins belong to a large supergene family of ubiquitous glycosyltransferases (GT) [23] (currently >10,000, classified into 87 subfamilies). Diverse members of the GT superfamily are noted for their low sequence similarity but, surprisingly, belong to only two structural folds (GT-A and GT-B) [24].

Glucuronidation is also an important pathway for biotransformation and clearance of drugs, such as colorectal cancer drug irinotecan [25-27]. Genetic polymorphisms or mutations in human UGT genes have profound impacts on hyperbilirubinemia, drug metabolism, and cancer treatment [20,27-29]. For example, mutations of the human UGT1A1 gene cause genetic diseases with phenotypes ranging from mild jaundice to lethal kernicterus $[20,28,30,31]$.

The human UGT1 cluster has an unusual genetic structure (Fig. 1A) which is strikingly similar to that of the Pcdh clusters [1]. Specifically, the human UGT1 cluster is organized into variable and constant regions [1,21,29,32,33]. About a dozen very similar human, mouse, and rat UGT1 variable exons (divided into bilirubin and phenol groups) are organized in a tandem array, which are followed by four constant exons (Fig. 1A) [1,33,34]. Splicing of each variable exon to the four constant exons generates diverse functional UGT1 mRNAs. Each variable exon encodes a signal peptide and the N-terminal aglycone-recognition domain of a UGT1 protein. The four constant exons encode the common C-terminal domain that binds the UDP glucuronic acid (UDPGA) donor substrate and an ER-anchoring transmembrane segment.

The role of the UGT genes in metabolizing myriad xenoand endobiotic compounds suggests that natural selection may have played an important role in shaping their variation; however, the effects selection might have on such unusual genomic structures are unclear. To gain insight into the evolution of multiple variable first exons, we annotated the complete vertebrate Ugt1, Gcnt2, and Ugt $2 a$ repertoires and identified 65 (for mRNA and protein sequences see Additional file 1), 16 (Additional file 2 ), and 16 (Additional file 3) new genes, respectively. Phylogenetic analyses on these clusters revealed lineagespecific duplications of variable exons and conservation of constant exons. Our results suggest that functional diversity of these clusters is achieved through the birth- 

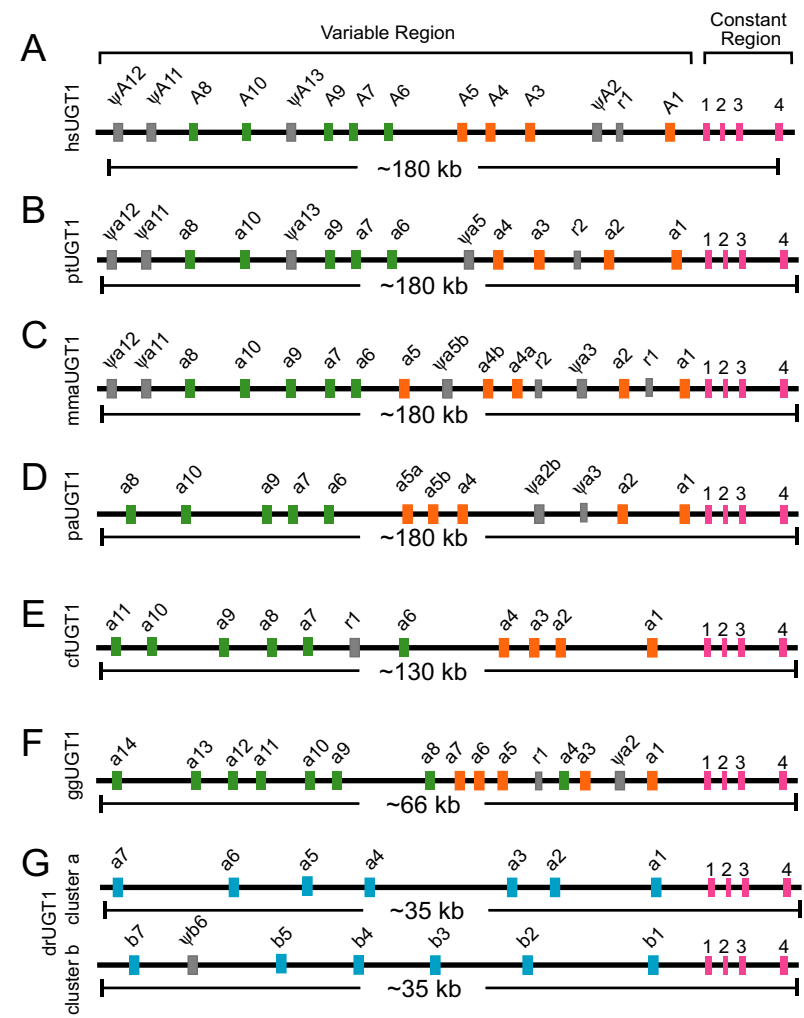

\section{Figure I}

Comparison of the $(\mathbf{A})$ human (Homo sapiens [hs]), (B) chimpanzee (Pan troglodydes [pt]), (C) rhesus monkey (Macaca mulatta [mma]), (D) baboon (Papio anubis [pa]), (E) dog (Canis familiaris [cf]), (F) chicken (Gallus gallus [gg]), and (G) zebrafish (Danio rerio [dr]) Ugt I clusters. Each cluster contains multiple variable first exons arrayed in tandem and a common set of 4 downstream constant exons. These exons are indicated by vertical colored bars: (green) phenol-group variable exons; (orange) bilirubin-group variable exons; (blue) zebrafish variable exons; (gray) pseudogene $(\psi)$ or relic $(r)$; and (red) constant exons. The approximate length of each cluster is shown below the corresponding panels.

and-death evolution of variable exon duplication, divergence, and deletion, but conservation of the constant exons, which are essential in maintaining their basic functions. In addition, analyzing the complete repertoires of closely-related $U g t 2 b$ clusters in humans, mice, and rats identified a new rat $U g t 2 b$ gene (designated Ugt2b39; Additional file 3 ) and revealed extensive lineage-specific duplications.

To gain insight into the catalytic mechanisms of glucuronidation by diverse UGT glucuronosyltransferases, we sought evidence for structural features in donor and acceptor recognition by combined comparative analysis and homologous modeling [35]. We built the first three- dimensional (3D) structure model of the vertebrate UGT proteins based on sequence analyses of 91 UGT1 and 35 UGT2 GT-B proteins, and the known crystal structures of the non-vertebrate GT-B glycosyltransferases. Molecular docking of donor and acceptor ligands to the human UGT1A1 structure shed light on the specificity of the donor recognition and diversity of acceptor bindings. In particular, we identified four hypervariable regions within the N-terminal domain that form a potential acceptorbinding pocket. We also identified Ugt codon sites that may have been subject to Darwinian positive selection during vertebrate evolution by analyzing patterns of nucleotide (nt) substitutions at individual codon sites. Interestingly, the diversified residues in the four hypervariable regions map to an acceptor-binding pocket. These residues likely contribute to the required specificity for binding numerous hydrophobic small molecules. These results suggest that adaptive natural selection of specific codon sites plays an important role for enhancing UGT diversity. In summary, our results provide insight into the evolution of multiple variable exons and structural diversity of UGT proteins required for the removal of numerous xenobiotic compounds and endogenous metabolites.

\section{Results and Discussion The vertebrate Ugt I gene cluster}

We analyzed Ugt1 locus in a set of diverse vertebrate species including primates, non-primate mammals, birds, and fish (Fig. 1 and Additional file 1). Chimpanzees are the closest living relatives of humans, and their genomic sequences are highly similar to those of humans. We found that two Ugt1 genes differ between humans and chimpanzees (Fig. 1A and 1B) although these species only diverged as recently as several million years ago [36]. The chimpanzee Ugt1a2 has a complete open reading frame suggesting being a functional gene while the human UGT1A2 has a single-nt deletion at coding position 127 causing a frameshift. In contrast, the human UGT1A5 is a functional gene while the chimpanzee Ugt1a5 appears to be a pseudogene because its sequences have a single-nt deletion at coding position 704. This frameshift deletion is confirmed by more than 10 different sequence reads.

We also annotated the rhesus monkey and baboon Ugt1 clusters (Fig. 1C and 1D). The Ugt1 clusters in these two old-world-monkey species contain one more functional variable exon than the human and chimpanzee Ugt1 clusters. Specifically, the bilirubin group (Ugt1 a1-a5) is expanded in these two species. Compared with humans and chimpanzees, the Ugt1a5 appears to have been duplicated to Ugt1a5a and Ugt1a5b in both rhesus monkey and baboon (Fig. 1C and 1D). The functional duplication of Ugt1a5 in baboon has also been reported in a very recent publication [37]. However, the Ugt1a5b has been mutated to a pseudogene in rhesus monkey because its sequences 
have a single-nt insertion in the coding region (Fig. 1C). In addition, the Ugt1a3 has been mutated to a pseudogene in both rhesus monkey and baboon. Finally, the rhesus monkey Ugt1a4 variable exon appears to have been duplicated to Ugt1a4a and Ugt1a4b (Fig. 1C). Similarly, the baboon Ugt1a2 variable exon has also been duplicated; however, one duplicated copy has been mutated to a pseudogene Ugt1a2b (Fig. 1D). Interestingly, the rhesus monkey Ugt1a 7 in the whole-genome-shotgun traces has no stop codon mutation; however, the Ugt1a7 in the finished BAC clone (Accession No. AC171066.4) has a stop codon at coding position 670. This observation suggests that Ugt1a7 has both functional and nonfunctional alleles segregating in the rhesus monkey population.

Dogs belong to the order Carnivora within the Laurasiatheria clade of mammals; while primates and rodents belong to the Euarchontoglire clade [38]. Dogs diverged from humans at about 94 million years ago while rodents diverged from primates at about 85 million years ago. The dog Ugt 1 cluster contains 10 functional variable exons (Fig. 1E). Members of the dog Ugt1 cluster can also be divided into the bilirubin and phenol groups. Compared with the primate Ugt1 cluster, the genomic region of the dog Ugt1 cluster is about $50 \mathrm{~kb}$ smaller (Fig. 1E).

The chicken separated from mammals about 310 million years ago [39]. Similar to the mammalian Ugt1 clusters, the chicken Ugt1 cluster is also organized into variable and constant regions and it has 14 variable exons arrayed in tandem, including one with frameshift mutations (Fig. 1F). Members of the chicken Ugt1 cluster can also be separated into bilirubin and phenol groups. The genomic region of the chicken Ugt1 cluster is much smaller than that of mammals (Fig. 1F).

The zebrafish has supernumerary $P c d h$ genes organized into two duplicated clusters $[4,5,7]$. Consistent with whole genome duplications in the teleost fish species and similar to the duplication of the zebrafish Pcdh clusters $[4,5,7]$, the zebrafish Ugt1 cluster has been duplicated into the Ugt1 $a$ and $b$ clusters each organized into variable and constant regions (Fig. 1G). In contrast to the vast expansion of the zebrafish Pcdh variable regions compared with mammals, the zebrafish Ugt1a and Ugt1b variable regions have not expanded. Specifically, compared with about a dozen Ugt1 variable exons in mammals, the zebrafish Ugt1a cluster only has seven functional variable exons, while the zebrafish Ugt1b cluster has only six functional variable exons (Fig. 1G). In total, we identified 13 novel zebrafish Ugt1 variable exons. Both zebrafish Ugt1a and $U g t 1 b$ clusters span a region of about $35 \mathrm{~kb}$ genomic sequences, much smaller than other vertebrate species analyzed.
The constant regions of mammalian, avian, and fish Ugt1 clusters are highly conserved and each contain 4 constant exons (Fig. 1). The length of each constant exon is identical among all vertebrate species except that the fourth constant exons are slightly smaller in frogs and zebrafish, encoding shorter polypeptides (Additional file 4). The two zebrafish constant sequences are highly similar with a $70 \%$ identity at the nt level and a $78 \%$ similarity at the polypeptide level. This observation strongly suggests that the two zebrafish Ugt1 clusters are duplicated from a single ancestral cluster. The polypeptides encoded by constant regions are highly conserved in vertebrates (Additional file 4).

\section{Evolutionary relationship among members of the vertebrate Ugt I clusters}

Similar to the Pcdh clusters [40], the variable region of each vertebrate UGT1 protein is encoded by a single unusually large exon (Fig. 1). These Ugt1 variable exons are similar and are of almost identical length with the same reading frame. Each human, mouse, and rat Ugt1 variable exon is preceded by a distinct promoter $[1,20,21,32,33]$. Consistently, there is a highly-conserved sequence motif upstream from each vertebrate Ugt1 variable exon (Additional file 5), suggesting that these conserved promoter motif sequences play a role in tissue-specific Ugt1 gene regulation in vertebrates. The encoded variable polypeptides have the same predicted 3D domain structures (see below). Each variable polypeptide consists of a signal sequence followed by an aglycone-recognition domain. Their evolutionary relationships are shown as an unrooted phylogenetic tree (Fig. 2). In conjunction with the clustered genomic organization (Fig. 1), the tree demonstrates that variable exons of the Ugt1 clusters are duplicated in tandem. Some members are maintained, while others are inactivated by deleterious mutations in specific vertebrate lineages, suggesting that birth-and-death evolution has occurred in the Ugt1 cluster.

The mammalian and avian Ugt1 clusters can be divided into two major groups (constant-proximal bilirubin group and constant-distal phenol group) (Fig. 1). These groups each have a long major branch while members within each group have relatively shorter secondary branches in the phylogenetic tree, suggesting that members within each group were duplicated recently (Fig. 2). The human, chimpanzee, baboon, rhesus monkey, and dog Ugt1a1 is orthologous. However, there is no obvious orthologous Ugt1a1 in the chicken Ugt1 cluster, suggesting that the specialization of bilirubin glucuronidation by Ugt1a1 occurs after the divergence of mammals and birds. Interestingly, the mammalian Ugt1a6 is orthologous and is remotely similar to three avian Ugt1 variable exons ( $a 4$, $a 8$, and $a 9$ ). This observation indicates that Ugt1a6 is more ancient than other Ugt1 members. 


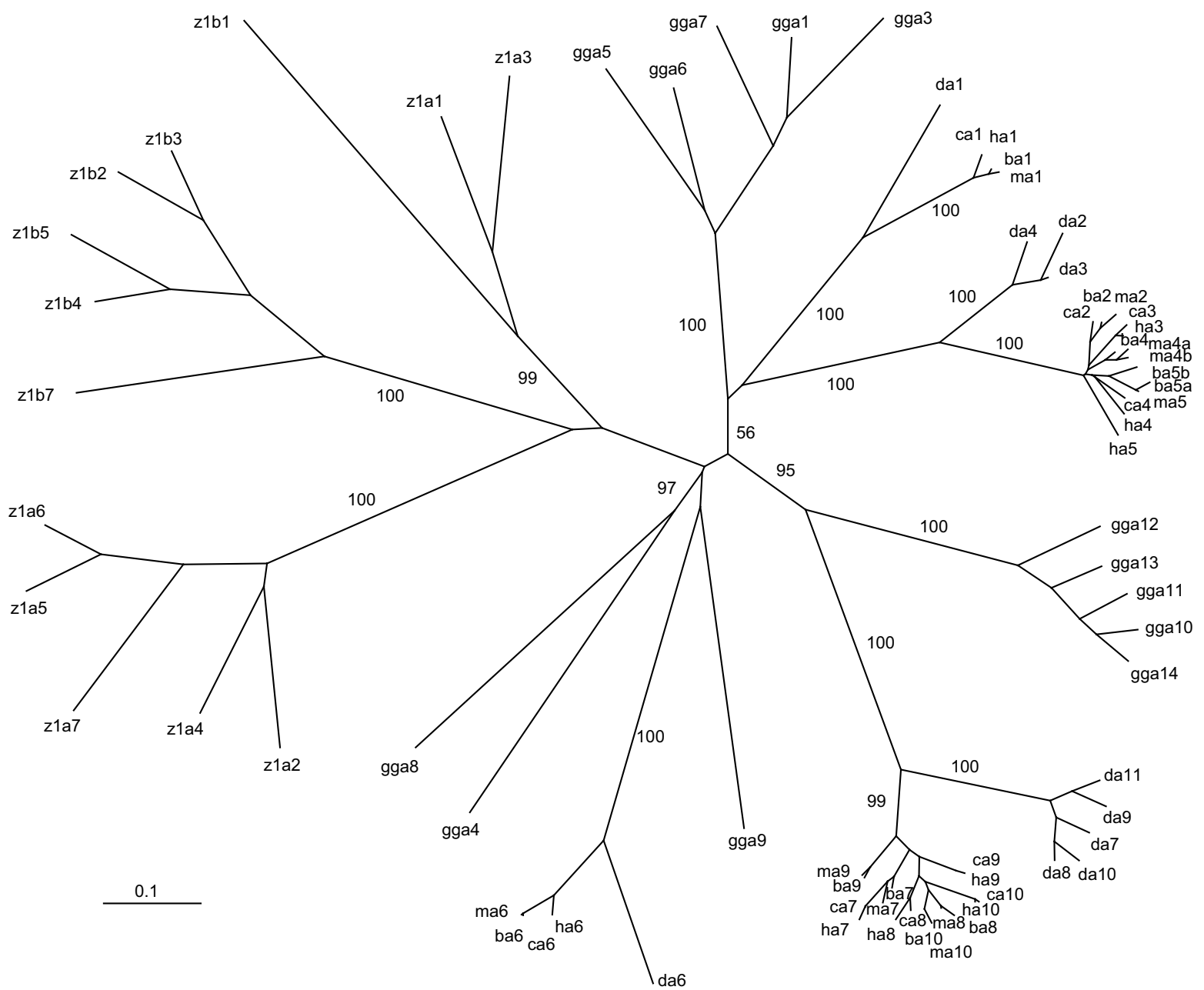

\section{Figure 2}

Phylogenetic tree of human (h), chimpanzee (c), rhesus monkey (m), baboon (b), dog (d), chicken (Gallus gallus [gg]), and zebrafish (z) Ugt I clusters. The major tree branches are labeled with the percentage support (only when $>50 \%$ ) for that partition based on I,000 bootstrap replicates. The scale bar equals a distance of 0.1 .

Members of the zebrafish Ugt1 clusters do not display orthologous relationships to those of the mammalian and avian Ugt1 clusters. Instead, they display paralogous relationships in one major branch of the phylogenetic tree (Fig. 2). They can be divided into three subgroups: subgroup 1 includes $z 1 a 1, z 1 a 3$, and $z 1 b 1$, subgroup 2 includes $z 1 b 2-b 7$, and subgroup 3 includes $z 1 a 2$ and $z 1 a 4$ $a 7$. The zebrafish Ugt1 $a$ and $b$ variable exons and the corresponding constant exons seem to have resulted from a duplication of an ancestral one-variable Ugt1 gene. Subsequently, the variable exons in each cluster are duplicated multiple rounds. For example, the zebrafish Ugt1 a1 and $a 2$, and $a 3$ and $a 4$ seem to be duplicated from an ancestral two-variable-exon unit because $a 1$ and $a 3$, and $a 2$ and $a 4$ share more sequence similarity, respectively. However, other zebrafish Ugt1 $a$ and $b$ variable exons seem to be duplicated in tandem because neighboring ones are more similar to each other.

Gene conversion plays an important role in the evolution of supergene families. Tandem gene arrays are often subject to sequence homogenization through gene conversion. For example, tandem arrayed Pcdh variable exons undergo strikingly predominant gene conversion events, especially among physically close exons [4]. To determine whether gene conversion played a similar prominent role in the evolution of vertebrate Ugt1 clusters, we used the Geneconv program [41] to search for gene conversion events among Ugt1 variable exons. Surprisingly, we did not find prevalent gene conversion events in the vertebrate Ugt1 clusters, except in the dog Ugt1 locus where gene conversion events have occurred in the phenol sub- 


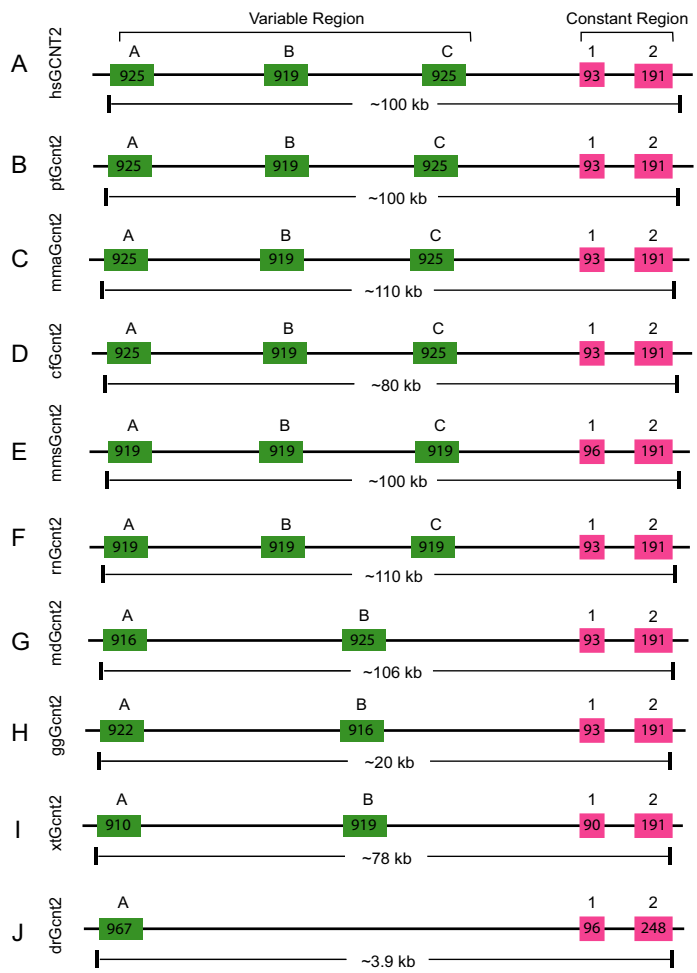

\section{Figure 3}

Comparison of the (A) human (Homo sapiens [hs]), (B) chimpanzee (Pan troglodydes [pt]), (C) rhesus monkey (Macaca mulatta [mma]), (D) dog (Canis familiaris [cf]), (E) mouse (Mus musculus [mms]), (F) rat (Rattus norvegicus [rn]), (G) opossum (Monodelphis domestica [md]), (H) chicken (Gallus gallus [gg]), (I) frog (Xenopus tropicalis [xt]), and (J) zebrafish (Danio rerio [dr]) Gcnt2 clusters. Each cluster contains multiple-variable and highly-similar first exons (green boxes) arrayed in tandem and a common set of two downstream constant exons (red boxes). Exon length is indicated within each box. The approximate length of each cluster is shown below the corresponding panels.

group (Ugt1 a7-a11) (Additional file 6). Consistently, no gene conversion event was detected between any two functional baboon genes [37]. This observation suggests that, in striking contrast to the Pcdh clusters, concerted evolution does not play a predominant role in the evolvement of the vertebrate Ugt1 cluster.

\section{The organization and evolution of the vertebrate Gent2 cluster}

The human, mouse, and rat Gcnt2 (also known as IGnT) clusters each contain three highly similar variable exons and two constant exons [1] (Fig. 3A, 3E, and 3F). Each var- iable exon is preceded by a distinct promoter and is separately spliced to a set of two constant exons to generate functional Gont2 mRNA. We analyzed the vertebrate Gent2 clusters and found that the numbers of variable exons are different among mammals, birds, amphibians, and fishes (Fig. 3 and Additional file 2). The three Gcnt2 variable exons are conserved in chimpanzees and rhesus monkeys (Fig. 3B and 3C). They are also conserved in dogs, mice, and rats (Fig. 3D, 3E, and 3F). However, there are only two Gcnt 2 variable exons in the opossum genome (Fig. 3G). Thus, the three Gcnt2 variable exons are conserved in primates, canids, and rodents but not opossums (Fig. 3A-G). There are only two Gcnt2 variable exons in the chicken and frog genomes (Fig. 3H and 3I). Finally, there is only one Gcnt2 variable exon in zebrafish (Fig. 3J). These results suggest that the tetrapod Gont2 variable exons were expanded by tandem duplication during vertebrate evolution.

The vertebrate Gcnt2 variable exons are about the same length and are very similar to each other. The encoded polypeptides are highly conserved (Additional file 7). Each Gcnt2 variable domain has a hydrophobic transmembrane segment close to the $\mathrm{N}$-terminal (Additional file 7). They also contain six cysteine residues that are identical among all GCNT2 proteins (Additional file 7). An evolutionary tree was built according to the variable GCNT2 polypeptides (Additional file 8). The three Gcnt2 variable exons display orthologous relationships among all eutherian mammals. Interestingly, the two opossum Gcnt2 variable exons display a paralogous relationship and appear to be more similar to the eutherian Gcnt $2 b$ variable exons. The two chicken and frog Gcnt2 variable exons also display paralogous relationships and are divergent from the mammalian Gcnt2 variable exons. The single zebrafish Gcnt2 variable exon appears most closely related to the frog Gont2 variable exons. This result supports the hypothesis that the Gcnt2 variable exons have expanded in tetrapods through tandem duplications during vertebrate evolution. The genomic organization of the Gcnt2 constant region is highly conserved in vertebrates (Fig. 3). For example, the first constant exons of vertebrate Gcnt2 cluster are all $93 \mathrm{nts}$ in length except in mice, frogs, and zebrafish, which are 96, 90, and 96 nts, respectively. The encoded constant protein sequences are conserved and have three identical cysteine residues (Additional file 9).

\section{The vertebrate Ugt2 cluster}

We previously identified more than three thousand human genes with multiple first exons through a genomewide computational analysis; however, only the first exons of the PCDH and GCNT2 clusters are highly similar [1]. We have noted that the genomic organization of the human UGT2A cluster [42] is also similar to that of the 
UGT1, PCDH, and GCNT2 clusters. In particular, the Cterminal domains of the human UGT2A proteins are identical and are encoded by a set of five constant exons; by contrast, the N-terminal domains are similar and each is encoded by a single variable exon. However, in contrast to the human UGT1 cluster, the human UGT2A cluster only contains two variable exons which share $64 \%$ nt sequence identity. The variable and constant organizations of the Ugt $2 a$ cluster are conserved in human, mouse, and rat genomes (Additional file 10, panels A, B, and C). For example, the human, mouse and rat Ugt $2 a$ variable exons are similar and of the same length. We annotated the Ugt2a clusters in several additional mammalian species (Additional file 3) and found that the Ugt2a organization of two variable exons and five constant exons is conserved.

In contrast to the expansion of the mammalian Ugt1 clusters compared with zebrafish (Fig. 1), we found that the variable region of the zebrafish Ugt2a cluster is expanded in comparison to the mammalian variable regions and contains 4 novel variable exons (Additional files 3 and 10). Phylogenetic analysis demonstrates that the mammalian Ugt2 $a 1$ and $a 2$ variable exons display a strict orthologous relationship (Additional file 11). However, there is no orthologous relationship between mammalian and zebrafish Ugt2a variable exons. The four zebrafish Ugt2a variable exons appear to be duplicated in tandem, with the Ugt2 a3 and $a 4$ duplicated most recently (Additional file 11). Multiple sequence alignment demonstrates that all vertebrate Ugt2a variable protein sequences are highly similar (Additional file 12). Like the constant region of the mammalian Ugt2a cluster, the zebrafish Ugt2a constant region contains 5 exons, which are highly similar to those of mammals. In particular, the sizes of constant exons 1 to 4 are identical between zebrafish and mammals, respectively. The zebrafish Ugt2a constant exon 5 coding region is $18 \mathrm{nts}$ longer than the corresponding mammalian Ugt2a constant exon (Additional file 10, panels $\mathrm{D}$ and $\mathrm{E}$ ). The polypeptides encoded by Ugt2a constant region are highly conserved in vertebrates (Additional file 13).

We performed a comprehensive analysis of the closelyrelated human, mouse, and rat Ugt $2 \mathrm{~b}$ genes and found one novel rat Ugt2b gene, designated Ugt2b39 (Additional file 10, panel C). The human, mouse, and rat Ugt $2 \mathrm{~b}$ genes are also clustered and are located very close to the Ugt2a cluster $[21,33]$. However, the genomic organizations of the human, mouse, and rat Ugt2b genes are different from the Ugt2a genes in that the Ugt2b genes do not share common constant exons. Each member of the Ugt $2 \mathrm{~b}$ cluster is an independent gene and contains six exons (Additional file 10). All corresponding exons are highly similar among members of the Ugt $2 \mathrm{~b}$ cluster. Their exon lengths are also identical among different mammalian species. The encoded UGT2B proteins are highly conserved among humans, mice, and rats. The transcription directions are the same for all members of the rat Ugt $2 b$ cluster, and are also the same for members of the rat Ugt $2 a$ genes. However, the transcription directions for members of the Ugt2b cluster are not the same in the human and mouse genomes (Additional file 10).

The evolutionary relationships of the Ugt2b genes are shown as an unrooted phylogenetic tree (Additional file $14)$. The human $U G T 2 B$ genes display paralogous relationships while members of the mouse and rat $U g t 2 b$ clusters display both paralogous and orthologous relationships. For example, the mouse Ugt2b1 and Ugt2b34 appears to be orthologous to the rat Ugt2b1 and Ugt2b34, respectively. However, the rat Ugt2b39 and Ugt2b34 genes appear to be duplicated from an ancestral gene because they are very similar and are also located next to each other (Additional file 10). The other mouse and rat $U g t 2 b$ genes do not have orthologous relationships. The phylogenetic tree suggests that most human, mouse, and rat $U g t 2 b$ genes are duplicated after speciation (Additional file 14).

In summary, we analyzed the Ugt1 loci in chimpanzee, rhesus monkey, baboon, dog, chicken, and zebrafish, and identified 65 new vertebrate Ugt1 genes (Additional file 1). Phylogenetic analysis demonstrated that the avian and mammalian Ugt1 variable regions are expanded compared to zebrafish (Figs. 1 and 2). We also performed a comprehensive analysis of the vertebrate Gont2 cluster and identified 16 new Gcnt2 genes (Additional file 2), and found that the variable region of the Gcnt2 cluster is also expanded during vertebrate evolution (Fig. 3). Finally, we analyzed the vertebrate Ugt2 repertoires and found that, in contrast to Ugt1 and Gcnt2 clusters, the zebrafish Ugt2a variable region has been expanded compared with mammals (Additional file 10). These results suggest that these vertebrate variable exons are subject to lineage-specific birth-and-death evolution.

\section{Structure modeling of the vertebrate UGT proteins}

The human UGT proteins allow our body to remove myriad endogenous metabolites and exogenous chemicals, such as steroids, bilirubin, bile acids, hormones, carcinogens, environmental toxicants, and therapeutic drugs $[20,26]$. Understanding their structures will shed light on the substrate specificity $[22,26]$. However, the 3D structure information, either based on X-ray data or molecular modeling, is not available to date. There are currently five crystal structures of the GT-B family members (MurG [43], GtfB [44], GtfA [45], GtfD [46], and UGT71G1 [47]). These structures are related although their primary sequences are divergent $[24,47]$. To comparatively model 


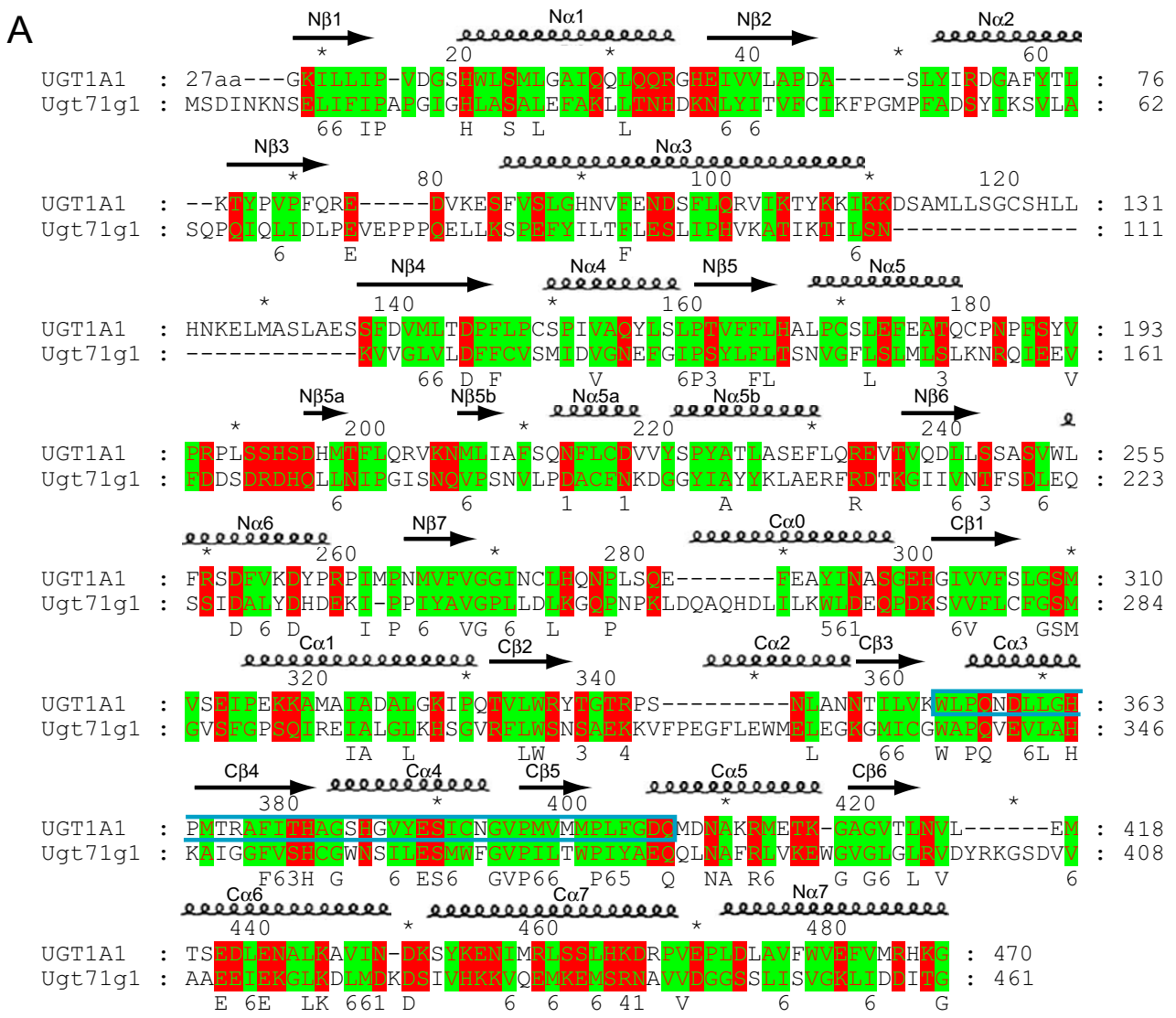

B

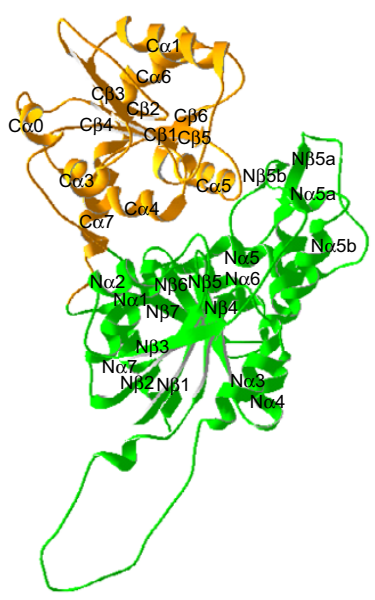

C



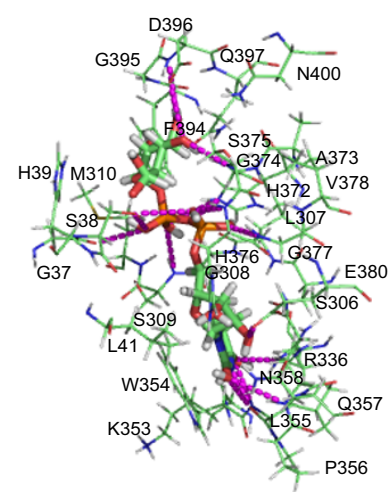

\section{Figure 4}

Modeling of the human UGTIAI protein. (A) Structural alignment of the human UGTIAI polypeptide with that of UGT7IGI. The secondary structure elements are shown above the alignment. The 44-aa donor signature motif of UGTIAI is enclosed by a cyan box. Broadly conserved hydrophilic and hydrophobic residues are highlighted with degree of conservation shown below the alignment. This panel was produced by the GeneDoc program [82]. (B) Ribbon diagram of the modeled 3D structure of the human UGTIAI. The $\mathrm{N}$ - and C-terminal domains are shown in green and orange, respectively. The $\alpha$ helices and $\beta$ strands in the $\mathrm{N}$ - and C-terminal domains are labeled. This panel was made by Swiss-PdbViewer [78]. (C) Stereo diagram showing predicted interactions between the donor UDPGA and the UGTIAI side chains. Hydrogen bonds are indicated by dashed lines. Figures 4C, 6B, 6C, and 8 were prepared with the Pymol [83]. 
vertebrate UGT protein structures, we first aligned the bacterial and plant GT-B polypeptides based on their 3D structures. We then aligned this structure-based alignment to the human UGT1A1 sequence based on the predicted vertebrate UGT secondary structure profile. We also aligned 91 vertebrate UGT1 and 35 human, mouse, rat, and zebrafish UGT2 polypeptides. Each of these translated 126 polypeptides has a signal peptide at the $\mathrm{N}$-terminal and a 17-amino-acid (aa) transmembrane segment close to the C-terminal with about 20 amino acids on the cytoplasmic side. The mature UGT proteins mostly reside in the lumen of the ER [22]. The structure of the human UGT1A1 within the ER lumen was modeled based on the alignment with UGT71G1 (Fig. 4A).

Our modeled 3D structure is consistent with that the vertebrate UGT proteins belong to the GT-B superfamily of the inverting glycosyltransferases [22,24]. Each modeled vertebrate UGT protein consists of two domains with similar core structure of Rossmann folds [48]. As an example, the modeled 3D structure of the human UGT1A1 protein is shown in Figure 4B. The N-terminal acceptor-binding domains of UGT1 proteins are each encoded by highlysimilar variable exons in all vertebrate species (Fig. 1). The C-terminal donor-binding domains of UGT1 proteins are identical in each species and are encoded by four constant exons (Fig. 1). For UGT2 proteins, the acceptor-binding domains are encoded by first two exons which correspond to a single Ugt1 variable exon, and the donor-binding domains are encoded by the last four exons [21]. The Cterminal domains of all vertebrate UGT proteins are highly conserved and assumed to bind the donor UDPGA [22].

The N-terminal acceptor-binding domain of the modeled human UGT1A1 contains a central seven-parallel-strand $\beta$-pleated sheet with a topological arrangement of $\beta 3, \beta 2$, $\beta 1, \beta 4, \beta 5, \beta 6, \beta 7$ (Fig. 4B). This core $\beta$ sheet is flanked by $8 \alpha$ helices. The first three $\beta$ strands are connected by two $\alpha$ helices (arranged in $\alpha 2$ and $\alpha 1$ orientation) on the same side of the $\beta$ sheet as the N $\alpha 7$ helix, which is from the Cterminal sequences but is located below the last four $\beta$ strands in the $\mathrm{N}$-terminal domain. The other side of the core $\beta$ sheet contains five helices with a topological arrangement of $\alpha 3, \alpha 4, \alpha 5 b, \alpha 5, \alpha 6$. Similar to the structure of UGT71G1, there is a small two-stranded $\beta$ sheet following the N $\alpha 5$ helix. In contrast to the structure of UGT71G1, there is a flexible loop and a small predicted $\alpha$ helix following the N $\alpha 3$ helix (Fig. 4B). This segment is predicted to have different conformations among different human UGT proteins.

The C-terminal donor-binding domain contains a central six-parallel-strand $\beta$-pleated sheet with a topology arrangement of $\beta 3, \beta 2, \beta 1, \beta 4, \beta 5, \beta 6$ (Fig. 4B). This $\beta$ sheet core is flanked by $7 \alpha$ helices with a topological arrangement of $\alpha 0, \alpha 3, \alpha 4, \alpha 5$ at one side of the $\beta$ sheet and $\alpha 1$ and $\alpha 6$ at the other side of the $\beta$ sheet, and $\alpha 7$ at the bottom. In contrast to UGT71G1, the human UGT1A1 does not appear to have the $\mathrm{C} \alpha 2$ helix. The last C-terminal $\alpha$ helix (N $\alpha 7$ ) is located at the bottom of the N-terminal domain. The two loops between $\mathrm{N} \beta 7$ and $\mathrm{C} \alpha 0$ and between $\mathrm{C} \alpha 7$ and $\mathrm{N} \alpha 7$ connect the $\mathrm{N}$-terminal and C-terminal domains (Fig. 4B).

\section{Interactions between UGT proteins and the donor substrate}

The donor substrate UDPGA for vertebrate UGT enzymes is predicted in our $3 \mathrm{D}$ model to bind in a long narrow channel mainly in the C-terminal Rossmann-fold domain (Fig. 4B and 4C). In particular, the donor sits in a groove formed by the N-terminal half of the C $\alpha 3$ and $\mathrm{C} \alpha 4$, and the C-terminal half of the $\mathrm{C} \beta 4$ and $\mathrm{C} \beta 5$. In the modeled donor-UGT1A1 complex, the uracil ring of UDPGA interacts with the side chain of R336 and the main chain of L355 and Q357 through hydrogen bonds, and also forms parallel stacking interaction with the indole ring of W354 of the human UGT1A1. The ribose ring of UDPGA interacts with the side chain of Q357, N358, and E380 through hydrogen bonds. The $\alpha$-phosphate forms hydrogen bonds with the side chain of S38 and H372 and the main chain of $\mathrm{H} 376$ and G377, while the $\beta$-phosphate interacts with the side chain of S38 and H372 and the main chain of S309 and G37. Finally, the glucuronic acid moiety interacts with the side chain and main chain of D396, the main chain of S375, and the side chain of Q397 through hydrogen bonds (Fig. 4C). Overall, the donor binding mode is similar to that observed in the complex of donor substrates with the GtfB, GtfD, MurG, and UGT71G1 proteins [43,45-47]. In addition, this model of the donor recognition is consistent with the crucial role of the human UGT1A6 histidine, arginine, aspartic, and glutamic residues as demonstrated by chemical modification and sitedirected mutagenesis experiments [22].

The sequences of donor-binding region are highly conserved, especially for the donor-interacting residues. For example, the residues interacting with UDPGA are identical among all vertebrate UGT1 proteins and are almost identical among the UGT2 proteins (Fig. 5). In addition, the residues located very close to UDPGA are also almost identical among the UGT1 and UGT2 proteins (Fig. 5). Previous studies have predicted that UDPGA binds to this region of UGT proteins [22,49]. However, the D394 of UGT1A6 (corresponding to the D396 of UGT1A1) were predicted to interact with the uridyl moiety, an orientation different from the known GT-B donor complexes [43,45-47] and our modeled donor-UGT1A1 interactions (Fig. 4C). 


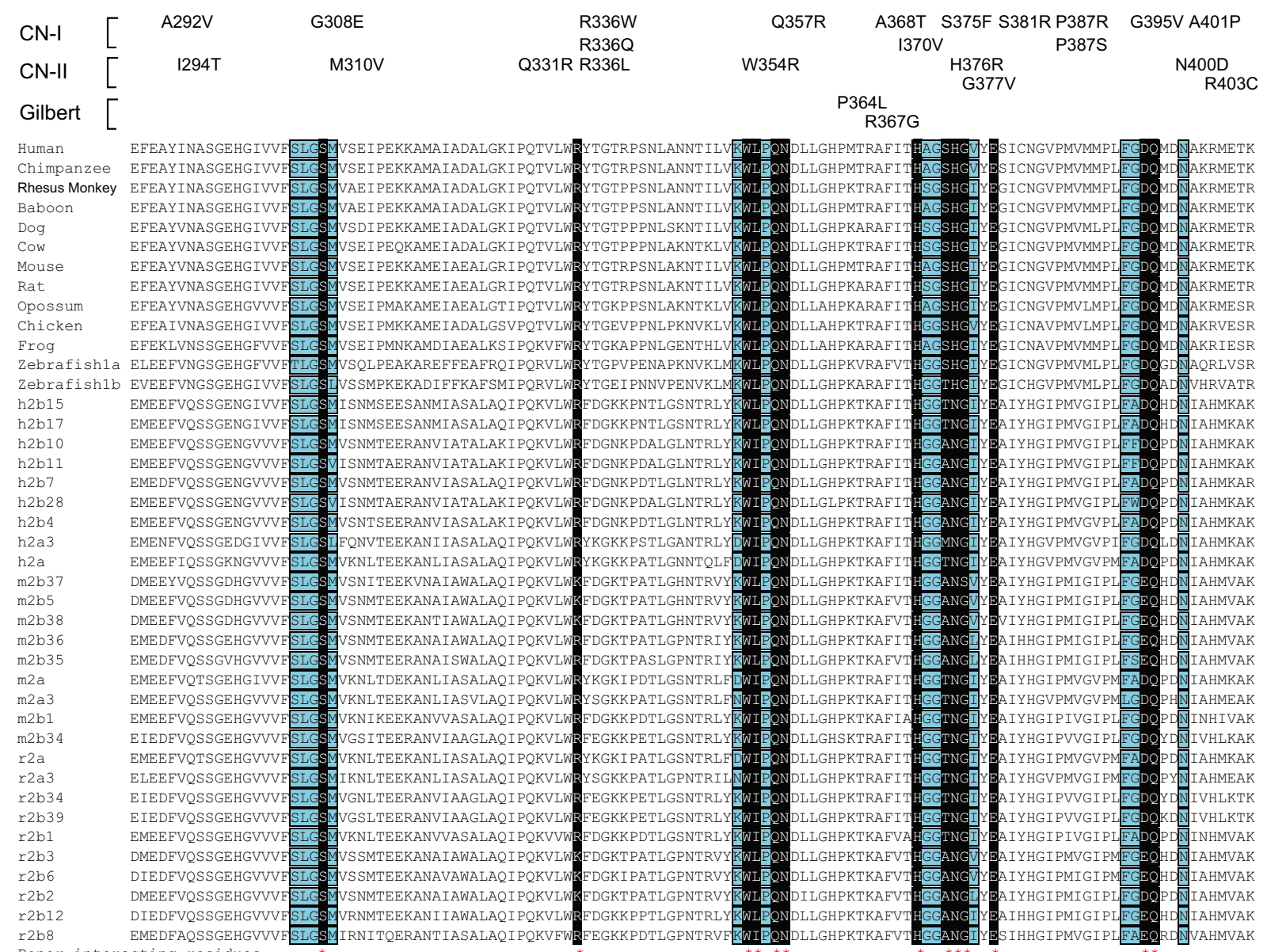

\section{Figure 5}

The donor-binding region of vertebrate UGT proteins. Shown is an alignment of the donor-binding region of the human, chimpanzee, rhesus monkey, baboon, dog, cow, mouse, rat, opossum, chicken, frog, and zebrafish UGTI constant polypeptides and the corresponding donor-binding region of the human $(\mathrm{h})$, mouse $(\mathrm{m})$, and rat $(\mathrm{r})$ UGT2A constant polypeptides and UGT2B proteins. Residues predicted to interact with the donor UDPGA are highlighted in white letters with black background and marked by red asterisks below. Residues close to the donor are highlighted with turquoise background and are also boxed. Missense mutations in the UGTIAI that cause human CN-I, CN-II, or Gilbert syndromes are indicated above the alignment. For UGTI, constant polypeptides are shared by multiple UGTIA proteins in each species. For UGT2, individual protein sequence is shown except h2a, m2a, and r2a, which are the human, mouse, and rat UGT2A constant polypeptides, respectively.

UGT proteins use UDPGA as a specific donor substrate [20]. In our modeled UDPGA UGT1A1 complex, the side chains of D396 and Q397 interact with the glucuronic acid moiety. These two residues may play an important role in the specific recognition of donor molecule by the UGT proteins. Consistently, Q397 is conserved in all vertebrate UGT1 and UGT2 proteins (Fig. 5). Similarly, D396 is conserved in all vertebrate UGT1 proteins and all human UGT2 proteins. It is also conserved in mouse and rat UGT2 proteins with a few replaced by a glutamic residue.
Missense mutations of human UGT1A1 cause hyperbilirubinemia, including type I and II Crigler-Najjar syndromes (CN-I, OMIM no. 218800 and CN-II, OMIM no. 606785 ) and the Gilbert syndrome (OMIM no. 143500) $[20,28,30]$. Point mutations with amino acid substitutions A292V (referred as A291V in [50]), G308E [50], R336W [51], R336Q [52], Q357R [50], A368T [50], I370V [53], S375F (referred as S376F in [54]), S381R [50], P387R [55], P387S [52], G395V [52], or A401P [50] cause the $\mathrm{CN}$-I disease (Fig. 5). Moreover, the missense substitutions I294T [51], M310V [56], Q331R [57], R336L [52], 


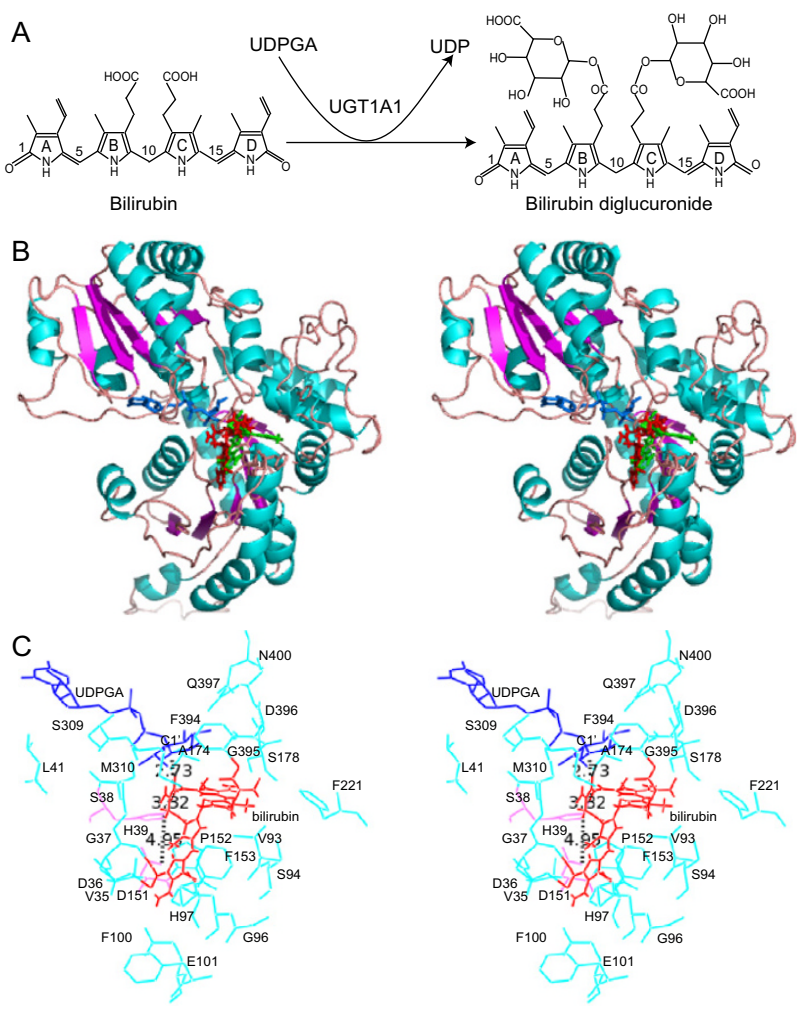

\section{Figure 6}

Molecular docking of bilirubin into the human UGTIAI protein. (A) Diagram of the bilirubin glucuronidation reaction catalyzed by UGTIAI. (B) Stereo diagram showing positions of the modeled substrates. The UGTIAI is shown in a ribbon diagram with the secondary structure highlighted. The donor UDP glucuronic acid (UDPGA) is shown as blue stick. The two docked conformations of bilirubin are shown as red and green sticks. (C) Stereo diagram showing bilirubin (red conformation) docked into the acceptor-binding pocket of UGTIAI. UDPGA (blue) and bilirubin (red) are shown as lines. Some residues in the acceptor-binding pocket are labeled and shown in cyan. Distances between the $\mathrm{OH}$ group of the bilirubin porphyrin $\mathrm{C}$ propionate and the $\mathrm{Cl}$ ' atom of the UDPGA or the NE2 atom of the residue histidine 39

$(\mathrm{H} 39)$ (shown in pink), or between the NE2 atom of the residue $\mathrm{H} 39$ and the OD2 atom of the residue aspartic acid I5I (DI5I) (shown in pink) are indicated with dashed lines.

W354R [52], H376R (referred as H377R in [28]), G377V [28], N400D [58] or R403C [52] cause the CN-II disease (Fig. 5). Finally, point mutations of P364L [59], or R367G $[60,61]$ cause the Gilbert syndrome (Fig. 5). These mutations are in the positions of highly conserved residues (with exception of only A292) in the donor-binding region (Figs. 4 and 5). In particular, the residues G308, M310, R336, W354, Q357, S375, H376, G377, S381, G395, N400, and A401 are located very close to the donor in the modeled 3D structure of human UGT1A1 protein
(Figs. 4C and 5). Their mutations may interfere with the donor binding, thus abolishing or decreasing the bilirubin glucuronidation activity of the human UGT1A1 protein and cause hyperbilirubinemia.

\section{The UGT acceptor-binding site}

Bilirubin is highly lipophilic, unexcretable, and neurotoxic, and is known to have a stable ridge-tile conformation [62]. The human UGT1A1 is the only UGT1 enzyme for glucuronidating bilirubin acceptor substrate [31]. It catalyzes the transfer of the glucuronic acid moiety from UDPGA to the bilirubin C8 and C12 propionate groups (Fig. 6A). In the modeled human UGT1A1 structure, there is a deep kinked pocket adjacent to the donor-binding site (Fig. 6B). This pocket is formed mostly by the N-terminal domain. Specifically, the wall of the pocket is formed by the $\mathrm{N} 1$ and $\mathrm{N} 2$ turns following the $\mathrm{N} \beta 1$ and $\mathrm{N} \beta 2$ strands,

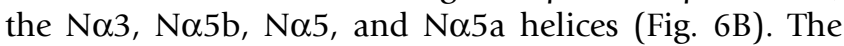
floor of the pocket is formed by the N $\beta 5$ strand and the $\mathrm{N} 4$ turn following the N $\mathrm{B} 4$ strand (Fig. 6B). The location of the pocket is similar to that of the substrate binding sites observed in the complex of the GtfA or GtfD with their corresponding acceptor substrates, and in the modeled acceptor-binding site of UGT71G1 [45-47]. Therefore, this pocket is likely the acceptor-binding site of the human UGT1A1. The glucuronic acid moiety of the donor in the modeled complex is oriented toward the middle of this acceptor-binding pocket.

We modeled the bilirubin binding using the molecular docking software GOLD [63]. The ridge-tile conformation of bilirubin is docked into the hydrophobic pocket with the ridge apposing the donor molecule in the C-terminal domain, and the porphyrin ring $\mathrm{A}$ in the one end and the porphyrin ring $\mathrm{D}$ in the other end of the $\mathrm{N}$-terminal acceptor pocket (Fig. 6B). The propionate side groups are in the middle and close to the glucuronic acid moiety of the donor molecules. Consistent with two glucuronidation sites in bilirubin through esterification of its two propionate side groups on the porphyrin rings $\mathrm{B}$ and $\mathrm{C}$, there are two conformations that the bilirubin docked in the acceptor pocket (Fig. 6B), with each propionate side group docked close to the glucuronic acid moiety of the donor and the highly conserved catalytic residue H39. There is a small tilt between the two bilirubin docking conformations. The acceptor-binding pocket is much larger and longer than bilirubin, and bilirubin can be fit easily into the pocket with one of its two propionate $\mathrm{OH}$ groups located at about 3 angstrom $(\AA)$ from the NE2 atom of the H39 residue (Fig. 6C).

The acceptor-binding pocket of the human UGT1A1 is surrounded by mostly hydrophobic residues in the N-terminal domain, including P34, V35, A64, L66, Y67, G71, F92, V93, G96, V99, F100, F153, F170, L172, A174, L175, 


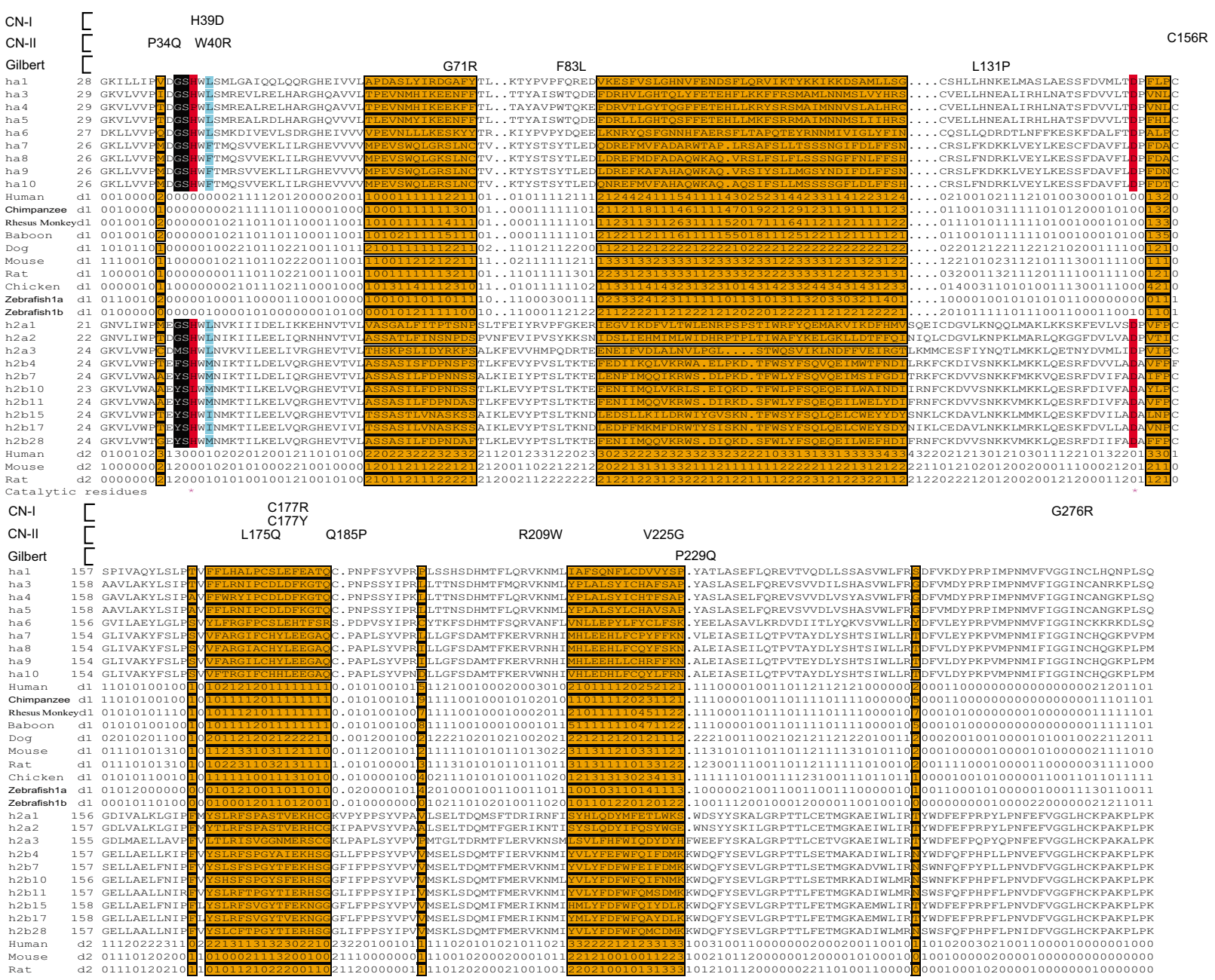

\section{Figure 7}

The acceptor-binding region of vertebrate UGT proteins. Shown is an alignment of the acceptor-binding region of the human UGTI and UGT2 proteins. The diversity indexes (defined as $\mathrm{K}_{\mathrm{A}} / \mathrm{K}_{\mathrm{S}}$ multiplied by 2 ) for the human, chimpanzee, rhesus monkey, baboon, dog, mouse, rat, chicken, and zebrafish UGTI (dI) and the human, mouse, and rat UGT2 (d2) are also shown. The four hypervariable regions (with exceptions of two cysteines) and several other highly diversified residues are highlighted in orange background and are also boxed. A leucine residue (L4I) close to the donor is highlighted in turquoise background. Residues predicted to interact with the donor molecule are highlighted with white letters in black background. Missense mutations in the human UGTIAI that cause CN-I, CN-II, or Gilbert syndromes are indicated above the alignment. The two catalytic residues histidine $39(\mathrm{H} 39)$ and aspartic acid I5I (DI5I) are highlighted in red background and with asterisks below.

F181, F221, V225, and A231. Interestingly, most of these hydrophobic residues are highly diversified among paralogous members of the vertebrate UGT proteins and are located within four major hypervariable regions in the $\mathrm{N}$ terminal domain (Fig. 7). These four hypervariable regions form the wall and floor of the acceptor-binding pocket in the N-terminal domain (Figs. 6B and 7). Analogous to the recognition of antigens by hypervariable regions of the IG, TCR, and MHC proteins, we propose that these hypervariable regions play an important role in the recognition of numerous aglycone-acceptor substrates by vertebrate UGT1 and UGT2 proteins.

Missense mutations of P34Q [52], H39D [28], W40R [64], G71R [61], F83L [65], L131P (referred as L132P in [61]), C156R [66], L175Q [67], C177R [67], C177Y [66], Q185P [64], R209W [67,68], V225G (referred as V224G in $[28,69]), P 229 Q[60,61]$, and G276R [67] in human UGT1A1 protein cause hyperbilirubinemia (Fig. 7). The P34, H39, and W40 residues are located in the acceptorbinding pocket and are also close to the donor substrate 
(Figs. 4, 6, and 7). The other mutated residues are mostly located in the four major hypervariable regions that form the acceptor-binding pocket. Therefore, these mutations may interfere with the binding of bilirubin to human UGT1A1 and abolish or decrease its bilirubin glucuronidating activity, consistent with the hyperbilirubinemia phenotypes. Interestingly, the UGT1A1 G71R mutation is almost exclusively found in Asians and has recently been shown to associate with severe cancer drug (i.e. irinotecan) toxicity [27], consistent with the altered acceptor recognition.

\section{Catalytic mechanism of vertebrate UGT glucuronidation}

Vertebrate UGT proteins belong to the GT-B inverting glycosyltransferase supergene family (Fig. 4) [22,24]. However, little is known about their catalytic mechanisms. In our modeled human UGT1A1 structure with the donor UDPGA and acceptor bilirubin substrates, the NE2 atom of the H39 residue lies in the middle of the potential acceptor pocket and is close to both the $\mathrm{OH}$ group of the bilirubin propionate side group $(\sim 3.32 \AA)$ and the $\mathrm{C} 1$ ' atom of UDPGA ( $2.73 \AA$ ), suggesting a general SN2 catalytic mechanism for glucuronidation reactions (Fig. 6C).

We propose that the H39 of human UGT1A1 acts as a general base to abstract a proton from the $\mathrm{OH}$ group of the bilirubin propionate, based on the crystal structures of other GT-B enzymes $[46,47]$. A direct attack by the resulting nucleophilic oxyanion at the $\mathrm{C} 1$ ' atom of UDPGA would then displace the UDP moiety. Consistent with the essential role of H39, it is highly conserved in vertebrate UGT proteins (Fig. 7). In addition, an H39D mutation in human UGT1A1 gene causes CN-I disease [28], consistent with the complete abolishment of the catalytic activity for bilirubin glucuronidation (Fig. 7).

In the modeled human UGT1A1 structure, there is an acidic D151 residue close to $\mathrm{H} 39$ that may form an electron transfer chain to help $\mathrm{H} 39$ deprotonate the $\mathrm{OH}$ group of the acceptor molecule (Fig. 6C). This aspartic acid residue is also highly conserved in vertebrate UGT proteins in agreement with its essential role in catalysis (Fig. 7). Thus, our modeled human UGT1A1 3D structure is consistent with genetic mutation data and provides a foundation for understanding the catalytic mechanism of vertebrate glucuronidation.

\section{Diversifying selection of vertebrate Ugt clusters}

Enormous molecular diversity is required for the immune and nervous system function. In the adaptive immune system, positive molecular selection operates to increase the diversity of the $I g, M h c$, and Tcr genes $[8,9,11,17,18,70]$. In the CNS, adaptive molecular selection also operates to enhance the diversity of the Pcdh and olfactory receptor gene clusters $[5,11,71]$. Human UGT proteins glucuroni-
A

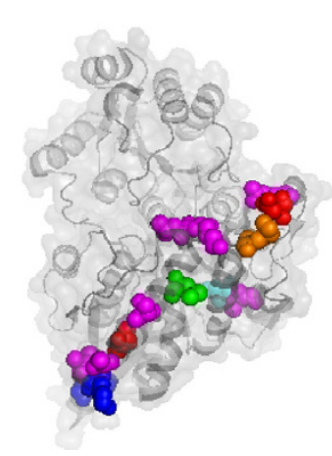

C



B
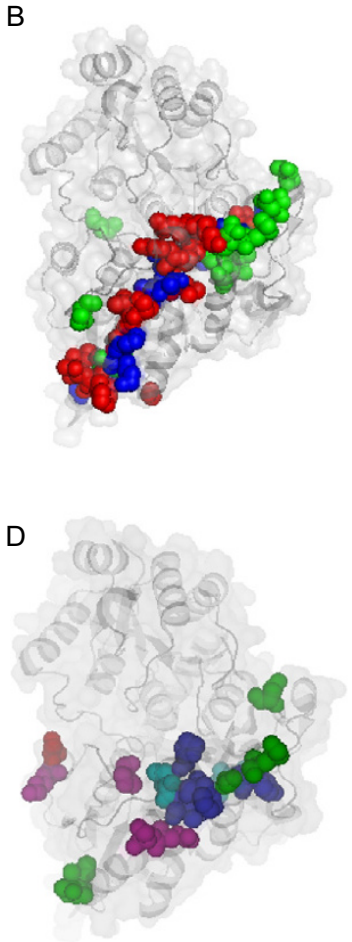

\section{Figure 8}

Site-specific $\mathrm{K}_{\mathrm{A}} / \mathrm{K}_{\mathrm{S}}$ analysis of the vertebrate Ugt I and Ugt2 clusters. Shown are positively selected $\omega+$ sites mapped to the acceptor domain of a ribbon diagram of the UGT7IG I crystal structure [47]. (A) Positively selected sites in the primate and dog UGTI. The $\omega^{+}$sites for the human only are highlighted in green; for the baboon only, in orange; for the rhesus monkey only, in cyan; for the dog only, in magenta; for the chimpanzee and dog only, in blue; and for all five species, in red. (B) Positively selected sites in the rodent UGTI. The $\omega+$ sites for the mouse only are highlighted in blue; for the rat only, in green; and for both mouse and rat, in red. (C) Positively selected sites in the chicken and zebrafish UGTI. The $\omega^{+}$sites for the chicken only are highlighted in blue; for the zebrafish cluster Ugt l $a$ only, in magenta; for the zebrafish cluster Ugt Ib only, in cyan; for both chicken and zebrafish cluster Ugt la, in red; and for both zebrafish clusters Ugt la and Ugt $l b$, in green. (D) Positively selected sites in the human, mouse, and rat UGT2. The $\omega+$ sites for the human only are highlighted in magenta; for the mouse only, in cyan; for the rat only, in green; for the mouse and rat only, in blue; and for all three species, in red. The residues mapped are comparably numbered according to the sequence of UGT7IGI and are equivalent between UGTI and UGT2, and among different vertebrate species. 
date numerous endogenous substrates including steroids and bile acids, as well as diverse xenobiotic chemicals such as environmental carcinogens and therapeutic drugs [20]. Gene duplication and birth-and-death evolution are major sources of UGT diversity in the vertebrate evolution (Fig. 1). We hypothesize that positive selection may be an additional factor to enhance the diversity of vertebrate Ugt genes.

We searched for positively selected sites in Ugt genes for various vertebrate species using the maximum-likelihood codeml program [72]. We ran three pairs of nested codeml models on the human, chimpanzee, rhesus monkey, baboon, dog, mouse, rat, chicken, and zebrafish clusters $a$ and $b$ Ugt1 genes, as well as the human, mouse, and rat Ugt2 genes to infer positively selected codon sites. The parameter estimates for the Ugt genes are shown in the Additional file 15 . The positively selected $\omega+$ sites in each repertoire are shown in the Additional file 16. Different vertebrate species have overlapping but distinct $\omega+$ site profiles for the Ugt1 and Ugt2 genes, even between very closely-related lineages such as mice and rats (Additional file 16), suggesting that these Ugt genes in different species are evolved through different chemical environments.

Based on the structural alignment in the Figure 4A, we aligned the polypeptide sequence of UGT71G1 to those of the human (Additional file 17), chimpanzee (Additional file 18), rhesus monkey (Additional file 19), baboon (Additional file 20), dog (Additional file 21), mouse (Additional file 22), rat (Additional file 23), chicken (Additional file 24), and zebrafish clusters A (Additional file 25) and B (Additional file 26) UGT1 variable-exonencoded polypeptides, as well as the corresponding human (Additional file 27), mouse (Additional file 28), and rat (Additional file 29) UGT2 proteins. We then mapped the positively-selected vertebrate UGT $\omega+$ sites onto the crystal structure of UGT71G1 [47] on the basis of these alignments (Fig. 8). Interestingly, almost all positively selected sites are located within the four vertebrate UGT hypervariable regions and map to the acceptor-binding pocket of the UGT71G1 crystal structure. Thus, these residues may participate in the recognition of diverse acceptor molecules by vertebrate UGT proteins. This observation suggests that nature selection operates to increase the diversity of vertebrate UGT proteins.

\section{Evolution of multiple variable first exons and UGT diversity}

We showed that the variable and constant organizations of Ugt1, Gcnt2, and Ugt2a clusters are vertebrate-specific (Figs. 1 and 3, and Additional file 10). In addition, these clusters are mainly subject to birth-and-death evolution instead of concerted evolution because there is no prevalent gene conversion (Additional file 6). Finally, nature selection at specific residues in four hypervariable regions in the UGT acceptor-binding domain increases their diversity for binding numerous environmental agents (Fig. 8 and Additional files 15 and 16). Interestingly, a recent human population genetic study found that diversified coding sites are more likely to be polymorphic than conserved sites [29].

In the vertebrate CNS, birth-and-death evolution of $P c d h$ variable exon arrays and positive selection on their specific ectodomain codons contribute to the staggering diversity required for neuronal connectivity [3-7]. In the vertebrate adaptive immune system, DNA rearrangement of variable and constant gene segments in the Ig and Tcr clusters, in conjunction with birth-and-death evolution and positive selection, generate unlimited diversity. Highly polymorphic Mhc genes also undergo birth-anddeath evolution and overdominant selection $[11,19]$. In particular, positive selection at hypervariable regions or CDRs of IG, TCR, and MHC proteins enhances their diversity for binding numerous antigens $[8-10,17,18]$. In the vertebrate detoxification system, UGT proteins recognize a myriad of hydrophobic aglycone molecules and each UGT has distinct but broad overlapping substrate specificities [20,49]. Similar to the nervous and immune systems, two factors contribute to the diversity of UGT proteins for defense against small chemicals. The duplication of Ugt1 variable exons and the entire Ugt2 genes increases the number of distinct vertebrate UGT proteins (Fig. 1 and Additional file 10). In addition, the diversified residues in hypervariable regions through positive selection contribute to the binding specificity of each vertebrate UGT protein for a large set of distinct aglycones (Figs. 7 and 8; Additional files 15 and 16). Thus, our results reveal an intriguing similarity of diversification mechanisms between vertebrate nervous, immune, and chemical defense systems.

\section{Conclusion}

The ability of UGT enzymes to glucuronidate numerous endobiotics and xenobiotics is conferred by their unusual genomic organization and structure diversity. Each Ugt1 variable exon is preceded by a distinct promoter. A highly conserved DNA motif located at about the same position upstream from each variable exon is likely to play an important role in regulating Ugt1 gene expression (Additional file 5). The combination of specific promoter activation and alternative cis-splicing of a variable exon to constant exons determines their tissue-specific expression. Comparative modeling of all UGT proteins suggests that each has di-domain Rossmann folds with a hydrophobic acceptor-binding pocket located within the N-terminal domain. Maximum-likelihood analysis of nt substitution patterns identified positively selected residues located in four hypervariable regions of the $\mathrm{N}$-terminal domain (Fig. 
7). Structural modeling suggests that these hypervariable regions form the hydrophobic acceptor-binding pocket (Fig. 6). Therefore, highly diversified residues in the acceptor-binding pocket could enable different UGT1 proteins to have distinct glucuronidation profiles for a large repertoire of environmental agents. Our comparative sequences analysis and homologous modeling shed light on the evolution of multiple variable exons and provide a framework for future structural and biochemical characterization of the vertebrate UGT proteins.

\section{Methods}

\section{Comparative sequence and phylogenetic analyses}

The vertebrate Ugt1 and Gint2, and mammalian Ugt2 genomic sequences were identified by iterative BLAST searches of the GenBank databases. The finished sequences were downloaded and analyzed as previously described $[1,5]$. The human gene nomenclature was following the recommendation of the HUGO committee. To ensure the accuracy, each nt was checked with the trace files from the TraceDB by using the Sequencher program. The sequences were analyzed for gene conversion by using the Geneconv program with default parameters [41]. Similar to previous convention [4], only sequence elements greater than $95 \mathrm{nt}$ in length shared among paralogs are shown. The variable Ugt1 and Gcnt2, and the full-length Ugt2 coding sequences were translated and the resulting polypeptides were aligned by using the GCG package. The promoter motifs were identified by the Gibbs sampler [73] and the graphic representations were generated by the Weblogo [74]. Phylogenetic trees were reconstructed by using the neighbor-joining algorithm in the ClustalW package. Gaps in the alignment were treated as missing during the tree construction. The robustness of the tree partitions was evaluated by bootstrap analyses.

\section{Homologous modeling of UGT structures and molecular docking of substrates}

We predicted the UGT1A1 secondary structure profile by using the neural network programs PSIPRED [75] and NNPREDICT [76], and aligned it to the structural alignment of known bacterial and plant GT-B crystal structures by using hidden Markov models (HMM) with manual adjustments [77]. We then modeled the structure of the human UGT1A1 by using the SWISS-MODEL [78]. The stereochemical quality of the structural model was evaluated with ANOLEA atomic mean force potential [79], GROMOS empirical force field energy, Verify3D profile [80], and the PROCHECK [81] programs. The modeled human UGT1A1 structure was refined by iterative modeling until there is no major difference in the active site between structural assessments of the model and the template [35]. In the final optimized UGT1A1 structure, dihedral angles of 331 residues were located in most favored regions of the Ramachandran plot, 53 residues in additional allowed regions, and 8 residues in generously allowed regions. We also modeled each of the 19 members of the human UGT1 and UGT2 families.

We modeled the UDPGA and bilirubin binding of UGT1A1 by using the molecular docking program GOLD (Genetic Optimization for Ligand Docking) [63] with default genetic algorithm parameters. The set up of the human UGT1A1 protein was according to the GOLD program manual. The UDPGA and bilirubin were downloaded from the PubChem Compound database. The UDPGA binding was modeled according to the cocrystal structure of UGT71G1 with the donor substrate. The bilirubin binding was modeled by seeding the atom NE2 of the residue $\mathrm{H} 39$ with a radius of $10 \AA$. GOLDscore was used to identify the lowest energy docking results. The hydrogen bonds and van der Waals interactions between ligands and UGT1A1 were analyzed to identify the optimal binding mode. The four hypervariable regions in the acceptor-binding domain were identified by multiple sequence alignment of all 91 vertebrate UGT1 variable polypeptides and the corresponding regions of 35 UGT2 proteins in conjunction with analyzing patterns of nt substitutions by the codeml program (see below).

\section{Site-specific $\mathbf{K}_{A} / \mathbf{K}_{S}$ analysis}

We used the maximum-likelihood codeml program of the PAML package (v3.15) [72] to predict codon sites under positive selection. The estimation of positively selected sites was performed as previously described [5]. Briefly, a set of 91 vertebrate Ugt1 variable exon sequences was translated and the resulting polypeptides were aligned with the N-terminal signal peptide removed. For the mammalian Ugt2 genes, a set of 31 full-length Ugt2 was aligned with both the N-terminal signal peptide and Cterminal transmembrane segment removed. The corresponding nt alignment was built by using RevTrans and separated into 10 Ugt1 (human, chimpanzee, rhesus monkey, baboon, dog, mouse, rat, chicken, and zebrafish clusters $a$ and $b$ ) and 3 Ugt2 (human, mouse, and rat) groups. For each of these 13 groups, we first ran the model $\mathrm{M} 0$ of the codeml program with a nt neighbor-joining tree to obtain a $\mathrm{K}_{\mathrm{S}}$-derived tree. By definition, the branches of the $K_{S}$ tree are about three times longer than those of the $n t$ tree. However, almost all of the $K_{S}$ values are $<1$, suggesting that synonymous substitutions are not saturated among these UGT paralogs. We then used this tree to run three nested pairs of codeml random-sites models: M0 vs. M3; M1a vs. M2a; and M7 vs. M8. Because iterative estimations of $\omega$ values by both M2a and M8 are susceptible to local optima, we ran these models with three different initial $\omega$ values $(0.03,0.8$, and 3.14$)$ and presented only those results with the highest likelihood. We mapped the positively selected $\omega+$ sites to the crystal structure of UGT71G1 (PDB accession code 2acv). The $\omega+$ sites were defined as diversified residues estimated to be under positive selection with a posterior probability of $>0.9$ by one 
codeml model (M2a, M3, or M8), and $>0.5$ by at least one other model $[5,71]$.

\section{Authors' contributions}

CL and QW performed experiments and analyzed data. QW conceived of the study and wrote the manuscript. All authors read and approved the final manuscript.

\section{Additional material}

\section{Additional file 1}

Vertebrate Ugt 1 genes. The mRNA and protein sequences for 65 new vertebrate Ugt 1 genes are shown in the FASTA format.

Click here for file

[http://www.biomedcentral.com/content/supplementary/14712148-7-69-S1.txt]

\section{Additional file 2}

Vertebrate Gcnt2 genes. The mRNA and protein sequences for 16 new and 9 known vertebrate Gcnt2 genes are shown in the FASTA format. Click here for file

[http://www.biomedcentral.com/content/supplementary/14712148-7-69-S2.txt]

\section{Additional file 3}

Vertebrate Ugt2a and the rat Ugt2b39 genes. The mRNA and protein sequences for 16 vertebrate Ugt2a and the rat Ugt2b39 genes are shown in the FASTA format.

Click here for file

[http://www.biomedcentral.com/content/supplementary/14712148-7-69-S3.txt]

\section{Additional file 4}

Alignment of vertebrate UGT1 constant polypeptides with conserved residues highlighted. The predicted 17-aa transmembrane segment across the $E R$ is marked by a line below. Identical conserved residues are shown in black box shade, similar conserved residues in grew shade, and nonidentical residues are left with a white background. Abbreviations for species: $M M s$, Mus musculus; RN, Rattus norvegicus; $M M a$, Macaca mulatta; $P A$, Papio anubis; HS, Homo sapiens; $P T$, Pan troglodytes; $C F$, Canis familiaris; $B T$, Bos taurus; $M D$, Monodelphis domestica; $G G_{,}$ Gallus gallus; XT, Xenopus tropicalis; and DR, Danio rerio, which has two highly similar constant polypeptides each is 6-aa shorter than in other vertebrate species.

Click here for file

[http://www.biomedcentral.com/content/supplementary/14712148-7-69-S4.pdf]

\section{Additional file 5}

Alignment of conserved sequence motifs upstream of the human $(h)$, chimpanzee (c), rhesus monkey (Macaca Mulatta [mma]), baboon (b), $\operatorname{dog}(d)$, mouse $(\mathrm{m})$, rat (r), chicken (Gallus gallus [gg]), and zebrafish $(z)$ Ugt1 variable exons. Shown are the conserved sequence motifs identified by the Gibbs sampler [73] in the 500 nt regions upstream of the translation start codon (indicated by the negative numbers flanking the motifs) of each Ugt 1 variable exon in the mammalian and avian bilirubin (A) and phenol (B) groups as well as in the zebrafish Ugt 1a and Ugt1b clusters $(E)$. The probability of the motif element is shown within parentheses on the right. The graphic sequence log representations [74] of the corresponding motifs are shown in panels $(C),(D)$, and $(F)$, respectively. The height of symbols corresponds to the relative frequency of each $\mathrm{nt}$. Click here for file

[http://www.biomedcentral.com/content/supplementary/14712148-7-69-S5.pdf]

\section{Additional file 6}

Gene conversion events in vertebrate multiple-variable-exon gene clusters. BC KA P-value: Bonferroni-corrected Karlin-Altschul P value. Only lengths $>95$ nt are shown.

Click here for file

[http://www.biomedcentral.com/content/supplementary/1471-

2148-7-69-S6.pdf]

\section{Additional file 7}

Alignment of vertebrate $G C N T 2$ variable polypeptides ( $A, B$, and $C)$ with conserved residues highlighted. The predicted transmembrane segment is marked by a line below. The six identical cysteine residues are marked by asterisks below. Identical conserved residues are shown in black box shade, similar conserved residues in grew shade, and nonidentical residues are left with a white background. Abbreviations for species: GG, Gallus gallus; XT, Xenopus tropicalis; MMs, Mus musculus; $R N$, Rattus norvegicus; PT, Pan troglodytes; $H S$, Homo sapiens; $M M a$, Macaca mulatta; $C F$, Canis familiaris; $M D$, Monodelphis domestica; and $D R$, Danio rerio.

Click here for file

[http://www.biomedcentral.com/content/supplementary/14712148-7-69-S7.pdf]

\section{Additional file 8}

Phylogenetic tree of the human (h), chimpanzee (c), rhesus monkey (Macaca mulatta [mma]), dog (d), mouse ( $\mathrm{m})$, rat ( $\mathrm{r})$, opossum (o), chicken (Gallus gallus $[\mathrm{gg}]$ ), frog $(f)$, and zebrafish (Danio rerio $[d r]$ ) Gont 2 clusters. The tree branches are labeled with the percentage support for that partition based on 1,000 bootstrap replicates. Only bootstrap values of $>50 \%$ on major branches are shown. The scale bar equals a distance of 0.1 .

Click here for file

[http://www.biomedcentral.com/content/supplementary/14712148-7-69-S8.pdf]

\section{Additional file 9}

Alignment of the vertebrate GCNT2 constant polypeptides with conserved residues highlighted. The three identical cysteine residues are marked by asterisks below. Identical conserved residues are shown in black box shade, similar conserved residues in grew shade, and nonidentical residues are left with a white background. Abbreviations for species: HS, Homo sapiens; PT, Pan troglodytes; $M M a$, Macaca mulatta; $C F$, Canis familiaris; $M M s$, Mus musculus; $R N$, Rattus norvegicus; $M D$,

Monodelphis domestica; GG, Gallus gallus; XT, Xenopus tropicalis; and $D R$, Danio rerio.

Click here for file

[http://www.biomedcentral.com/content/supplementary/14712148-7-69-S9.pdf] 


\section{Additional file 10}

Click here for file

[http://www.biomedcentral.com/content/supplementary/1471-

2148-7-69-S10.pdf]

\section{Additional file 11}

Phylogenetic tree of the human ( $h)$, chimpanzee (c), rhesus macaque (Macaca mulatta [mma]), dog (d), mouse $(\mathrm{m})$, rat $(\mathrm{r})$, and zebrafish (z) Ugt2a clusters. The major branches of the tree are labeled with the percentage support for that partition based on 1,000 bootstrap replicates. Only bootstrap values of $>50 \%$ on major branches are shown. The scale bar equals a distance of 0.1 .

Click here for file

[http://www.biomedcentral.com/content/supplementary/14712148-7-69-S11.pdf]

\section{Additional file 12}

Alignment of the vertebrate UGT2A variable polypeptides with conserved residues highlighted. The predicted signal peptides are indicated by a line below. Identical conserved residues are shown in black box shade, similar conserved residues in grew shade, and nonidentical residues are left with a white background. Abbreviations for species: DR, Danio rerio; MMs, Mus musculus; RN, Rattus norvegicus; PT, Pan troglodytes; HS, Homo sapiens; MMa, Macaca mulatta; and CF, Canis familiaris. Click here for file

[http://www.biomedcentral.com/content/supplementary/14712148-7-69-S12.pdf]

\section{Additional file 13}

Alignment of the vertebrate UGT2A constant polypeptides with conserved residues highlighted. The predicted 17-aa transmembrane segment across the ER is marked by a line below. Identical conserved residues are shown in black box shade, similar conserved residues in grew shade, and nonidentical residues are left with a white background. Abbreviations for species: MMs, Mus musculus; $R N$, Rattus norvegicus; $C F$, Canis familiaris; $P T$, Pan troglodytes; HS, Homo sapiens; $M M a$, Macaca mulatta; and $D R$, Danio rerio.

Click here for file

[http://www.biomedcentral.com/content/supplementary/14712148-7-69-S13.pdf]

\section{Additional file 14}

Phylogenetic tree of the human (h), mouse $(\mathrm{m})$, and rat $(r)$ Ugt2b clusters. The major tree branches are labeled with the percentage support for that partition based on 1,000 bootstrap replicates. The scale bar equals a distance of 0.1 .

Click here for file

[http://www.biomedcentral.com/content/supplementary/14712148-7-69-S14.pdf]

\section{Additional file 15}

Log-likelihood values and parameter estimates for human, chimpanzee, rhesus monkey, baboon, dog, mouse, rat, chicken, and zebrafish Ugt1 groups, and human, mouse, and rat Ugt 2 groups. Model ${ }^{1}$ Maximum-likelihood models implemented in the codeml program of the PAML package. $M 0$, one-ratio; $M 1 a$, neutral; $M 2 a$, selection; $M 3$, discrete; $M 7, \beta ; M 8$, $\beta+\omega . \ell^{2}$ Estimated log-likelihood values by the codeml program. $\kappa^{3}$ Estimated transition/transversion rate ratio by the codeml program. Estimation of Parameters ${ }^{4} \omega=K_{A} / K_{S}$ nonsynonymous/synonymous rate ratio; $p$ = proportion of sites for each site class. MO: one estimated $\omega$ for all sites; M1a: estimate $p_{0}=$ proportion of sites with $\omega_{0}=0, p_{1}=1-p_{0}$, proportion of sites with $\omega_{1}=1 ; M 2 a$ : estimate $p_{0}\left(\omega_{0}=0\right), p_{1}\left(\omega_{0}=1\right)$, and $\omega_{2}, p_{2}=$ $1-p_{0}-p_{1}$. M3: estimate $p_{0}, p_{1}, \omega_{0}, \omega_{1}$, and $\omega_{2} ; p_{2}=1-p_{0}-p_{1}$. M7: estimates $p$ and $q$ (parameters of $\beta$ distribution of $\omega$ between 0 and 1). M8: same as M7 except additional site class where an estimated $\omega$ is allowed. LRT $(2 \Delta \ell)^{5}$ Statistical likelihood ratio test; comparing the test statistic

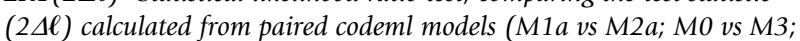
and $M 7 v$ s M8) with the critical value of chi-square asymptotic distribution with appropriate degrees of freedom (i.e. 2 d.f., 4 d.f., and 2 d.f., respectively). $2 \Delta \ell$ and level of significance are shown for $M 2 a, M 3$, and M8 models. Positively Selected Sites ${ }^{6}$ Codon positions predicted to be under positive selection with a posterior probability $>0.90 \mathrm{by}$ one codeml model (M2a, M3, or M8), and $>0.50$ by at least one other model. Click here for file [http://www.biomedcentral.com/content/supplementary/14712148-7-69-S15.pdf]

\section{Additional file 16}

Summary information for Ugt 1 and Ugt 2 groups analyzed. Tree length ${ }^{1}$ Measured as the number of nt substitutions along the tree per codon by the codeml program. The $\omega+$ sites $^{2}$ Codon positions predicted to be under positive selection with a posterior probability $>0.90$ by one codeml model (M2a, M3, or M8), and $>0.50$ by at least one other model. For Ugt 1 sequences, we used only the variable exons. For Ugt 2 sequences, we used full-length sequences but excluded Ugt $2 \mathrm{a} 2$ because it shares five constant exons with Ugt2a1.

Click here for file

[http://www.biomedcentral.com/content/supplementary/14712148-7-69-S16.pdf]

\section{Additional file 17}

Alignment of the protein sequences of UGT71G1 to those of the human (Additional file 17), chimpanzee (Additional file 18), rhesus monkey (Additional file 19), baboon (Additional file 20), dog (Additional file 21), mouse (Additional file 22), rat (Additional file 23), chicken (Additional file 24), and zebrafish clusters A (Additional file 25) and B (Additional file 26) variable UGT1 sequences, as well as the corresponding human (Additional file 27), mouse (Additional file 28), and rat (Additional file 29) UGT2 proteins. Each vertebrate UGT group was aligned by ClustalW and then aligned with UGT71G1 according to the structural alignment shown in the Figure 4A. The $\omega+$ sites predicted to be subject to positive selection with a posterior probability $>0.90$ by one model and $>0.5$ by at least one other model are highlighted in red.

Click here for file

[http://www.biomedcentral.com/content/supplementary/14712148-7-69-S17.pdf] 


\section{Additional file 18}

Click here for file

[http://www.biomedcentral.com/content/supplementary/14712148-7-69-S18.pdf]

\section{Additional file 19}

Click here for file

[http://www.biomedcentral.com/content/supplementary/1471-

2148-7-69-S19.pdf]

\section{Additional file 20}

Click here for file

[http://www.biomedcentral.com/content/supplementary/14712148-7-69-S20.pdf]

\section{Additional file 21}

Click here for file

[http://www.biomedcentral.com/content/supplementary/1471-

2148-7-69-S21.pdf]

\section{Additional file 22}

Click here for file

[http://www.biomedcentral.com/content/supplementary/14712148-7-69-S22.pdf]

\section{Additional file 23}

Click here for file

[http://www.biomedcentral.com/content/supplementary/1471-

2148-7-69-S23.pdf]

\section{Additional file 24}

Click here for file

[http://www.biomedcentral.com/content/supplementary/14712148-7-69-S24.pdf]

\section{Additional file $\mathbf{2 5}$}

\section{Click here for file}

[http://www.biomedcentral.com/content/supplementary/14712148-7-69-S25.pdf]

\section{Additional file 26}

Click here for file

[http://www.biomedcentral.com/content/supplementary/14712148-7-69-S26.pdf]

\section{Additional file 27}

Click here for file

[http://www.biomedcentral.com/content/supplementary/14712148-7-69-S27.pdf]

\section{Additional file 28}

Click here for file

[http://www.biomedcentral.com/content/supplementary/14712148-7-69-S28.pdf]

\section{Additional file 29}

Click here for file

[http://www.biomedcentral.com/content/supplementary/14712148-7-69-S29.pdf]

\section{Acknowledgements}

We would like to thank Drs. M. Leppert, J. Metherall, W. Sundquist, D. Witherspoon, S. Wooding, M. Yandell, and G. Ying for critical reading of the manuscript. Q.W. is a March of Dimes Basil O'Connor Scholar. Supported by an American Cancer Society Research Scholar Grant (RSG-03034-I-DDC) to Q.W.

\section{References}

I. Zhang T, Haws P, Wu Q: Multiple variable first exons: a mechanism for cell- and tissue-specific gene regulation. Genome Res 2004, 14:79-89.

2. Wu $\mathrm{Q}$, Maniatis $\mathrm{T}$ : A striking organization of a large family of human neural cadherin-like cell adhesion genes. Cell 1999, 97:779-790.

3. Wu Q, Zhang T, Cheng JF, Kim Y, Grimwood J, Schmutz J, Dickson M, Noonan JP, Zhang MQ, Myers RM, Maniatis T: Comparative DNA sequence analysis of mouse and human protocadherin gene clusters. Genome Res 200 I, I I:389-404.

4. Noonan JP, Grimwood J, Schmutz J, Dickson M, Myers RM: Gene conversion and the evolution of protocadherin gene cluster diversity. Genome Res 2004, I 4:354-366.

5. Wu Q: Comparative genomics and diversifying selection of the clustered vertebrate protocadherin genes. Genetics 2005 , 169:2179-2188.

6. Zou C, Huang W, Ying G, Wu Q: Sequence analysis and expression mapping of the rat clustered protocadherin gene repertoires. Neuroscience 2007, 144:579-603.

7. Tada MN, Senzaki K, Tai Y, Morishita H, Tanaka YZ, Murata Y, Ishii Y, Asakawa S, Shimizu N, Sugino H, Yagi T: Genomic organization and transcripts of the zebrafish Protocadherin genes. Gene 2004, 340: |97-2II.

8. Tanaka T, Nei M: Positive darwinian selection observed at the variable-region genes of immunoglobulins. Mol Biol Evol 1989, 6:447-459.

9. Sitnikova T, Nei M: Evolution of immunoglobulin kappa chain variable region genes in vertebrates. Mol Biol Evol 1998, 15:50-60.

10. Su C, Nei M: Evolutionary dynamics of the $T$-cell receptor VB gene family as inferred from the human and mouse genomic sequences. Mol Biol Evol 200I, I 8:503-5I3.

II. Nei M, Rooney AP: Concerted and birth-and-death evolution of multigene families. Annu Rev Genet 2005, 39:121-152.

12. Bjorkman PJ, Saper MA, Samraoui B, Bennett WS, Strominger JL, Wiley DC: Structure of the human class I histocompatibility antigen, HLA-A2. Nature 1987, 329:506-512.

13. Brown JH, Jardetzky TS, Gorga JC, Stern LJ, Urban RG, Strominger JL, Wiley DC: Three-dimensional structure of the human class II histocompatibility antigen HLA-DRI. Nature 1993, 364:33-39.

14. Bjorkman PJ, Saper MA, Samraoui B, Bennett WS, Strominger JL, Wiley DC: The foreign antigen binding site and $T$ cell recognition regions of class I histocompatibility antigens. Nature 1987, 329:512-518

15. Brown JH, Jardetzky T, Saper MA, Samraoui B, Bjorkman PJ, Wiley DC: A hypothetical model of the foreign antigen binding site of class II histocompatibility molecules. Nature I 988, 332:845-850.

16. Stern LI, Brown JH, Jardetzky TS, Gorga JC, Urban RG, Strominger JL Wiley DC: Crystal structure of the human class II MHC protein HLA-DRI complexed with an influenza virus peptide. Nature 1994, 368:215-221.

17. Hughes AL, Nei M: Pattern of nucleotide substitution at major histocompatibility complex class I loci reveals overdominant selection. Nature 1988, 335:167-170.

18. Hughes AL, Nei M: Nucleotide substitution at major histocompatibility complex class II loci: evidence for overdominant selection. Proc Natl Acad Sci U S A 1989, 86:958-962.

19. Nei M, Gu X, Sitnikova T: Evolution by the birth-and-death process in multigene families of the vertebrate immune system. Proc Natl Acad Sci U S A 1997, 94:7799-7806.

20. Tukey RH, Strassburg CP: Human UDP-glucuronosyltrans ferases: metabolism, expression, and disease. Annu Rev Pharmacol Toxicol 2000, 40:581-6I6

21. Mackenzie PI, Walter Bock K, Burchell B, Guillemette C, Ikushiro S lyanagi T, Miners JO, Owens IS, Nebert DW: Nomenclature update for the mammalian UDP glycosyltransferase (UGT) gene superfamily. Pharmacogenet Genomics 2005, I 5:677-685.

22. Radominska-Pandya A, Ouzzine $M$, Fournel-Gigleux S, Magdalou J: Structure of UDP-glucuronosyltransferases in membranes. Methods Enzymol 2005, 400: I I6-I47.

23. Coutinho PM, Deleury E, Davies G], Henrissat B: An evolving hier archical family classification for glycosyltransferases. J Mol Biol 2003, 328:307-317 [http://afmb.cnrs-mrs.fr/CAZY/fam/acc GT.html].

24. Hu Y, Walker S: Remarkable structural similarities between diverse glycosyltransferases. Chem Biol 2002, 9: I 287-I 296. 
25. lyer L, Hall D, Das S, Mortell MA, Ramirez J, Kim S, Di Rienzo A, Ratain MJ: Phenotype-genotype correlation of in vitro SN-38 (active metabolite of irinotecan) and bilirubin glucuronidation in human liver tissue with UGTIAI promoter polymorphism. Clin Pharmacol Ther 1999, 65:576-582.

26. Miners JO, Smith PA, Sorich MJ, McKinnon RA, Mackenzie PI: Predicting human drug glucuronidation parameters: application of in vitro and in silico modeling approaches. Annu Rev Pharmacol Toxicol 2004, 44: I-25.

27. Innocenti F, Vokes EE, Ratain MJ: Irinogenetics: what is the right star? J Clin Oncol 2006, 24:222I-2224.

28. Kadakol A, Ghosh SS, Sappal BS, Sharma G, Chowdhury JR, Chowdhury NR: Genetic lesions of bilirubin uridine-diphosphoglucuronate glucuronosyltransferase (UGTIAI) causing CriglerNajjar and Gilbert syndromes: correlation of genotype to phenotype. Hum Mutat 2000, 16:297-306.

29. Maitland ML, Grimsley C, Kuttab-Boulos H, Witonsky D, Kasza KE Yang L, Roe BA, Di Rienzo A: Comparative genomics analysis of human sequence variation in the UGTIA gene cluster. Pharmacogenomics / 2006, 6:52-62.

30. Crigler JF Jr., Najjar VA: Congenital familial nonhemolytic jaundice with kernicterus. Pediatrics 1952, 10:169-180.

31. Bosma PJ, Seppen J, Goldhoorn B, Bakker C, Oude Elferink RP, Chowdhury JR, Chowdhury NR, Jansen PL: Bilirubin UDP-glucuronosyltransferase $I$ is the only relevant bilirubin glucuronidating isoform in man. J Biol Chem 1994, 269: I7960-I7964.

32. Ritter JK, Chen F, Sheen YY, Tran HM, Kimura S, Yeatman MT, Owens IS: A novel complex locus UGTI encodes human bilirubin, phenol, and other UDP-glucuronosyltransferase isozymes with identical carboxyl termini. J Biol Chem 1992, 267:3257-326I.

33. Owens IS, Basu NK, Banerjee R: UDP-glucuronosyltransferases: gene structures of UGTI and UGT2 families. Methods Enzymol 2005, 400: I-22.

34. Emi $Y$, Ikushiro S, lyanagi T: Drug-responsive and tissue-specific alternative expression of multiple first exons in rat UDP-glucuronosyltransferase family I (UGTI) gene complex. J Biochem (Tokyo) 1995, I I 7:392-399.

35. Marti-Renom MA, Stuart AC, Fiser A, Sanchez R, Melo F, Sali A: Comparative protein structure modeling of genes and genomes. Annu Rev Biophys Biomol Struct 2000, 29:29|-325.

36. Chen FC, Li WH: Genomic divergences between humans and other hominoids and the effective population size of the common ancestor of humans and chimpanzees. Am J Hum Genet 200I, 68:444-456

37. Caspersen CS, Reznik B, Weldy PL, Abildskov KM, Stark RI, Garland $M$ : Molecular cloning of the baboon UDP-glucuronosyltransferase IA gene family: Evolution of the primate UGT I locus and relevance for models of human drug metabolism. Pharmacogenet Genomics 2007, I 7: I I-24.

38. Springer MS, Murphy WJ, Eizirik E, O'Brien SJ: Placental mammal diversification and the Cretaceous-Tertiary boundary. Proc Natl Acad Sci U S A 2003, I 00:1056-106I.

39. Hedges SB: The origin and evolution of model organisms. Nat Rev Genet 2002, 3:838-849.

40. Wu Q, Maniatis T: Large exons encoding multiple ectodomains are a characteristic feature of protocadherin genes. Proc Nat Acad Sci U S A 2000, 97:3124-3129.

41. Sawyer S: Statistical tests for detecting gene conversion. Mo Biol Evol 1989, 6:526-538 [http://www.math.wustl.edu/ sawyer/gene conv].

42. Tukey RH, Strassburg CP: Genetic multiplicity of the human UDP-glucuronosyltransferases and regulation in the gastrointestinal tract. Mol Pharmacol 200I, 59:405-4I4.

43. Hu Y, Chen L, Ha S, Gross B, Falcone B, Walker D, Mokhtarzadeh M, Walker S: Crystal structure of the MurG:UDP-GIcNAc complex reveals common structural principles of a superfamily of glycosyltransferases. Proc Natl Acad Sci U S A 2003, I 00:845-849.

44. Mulichak AM, Losey HC, Walsh CT, Garavito RM: Structure of the UDP-glucosyltransferase GtfB that modifies the heptapeptide aglycone in the biosynthesis of vancomycin group antibiotics. Structure 2001, 9:547-557.

45. Mulichak AM, Losey HC Lu W Wawrzak Z Walsh CT, Garavito RM: Structure of the TDP-epi-vancosaminyltransferase GtfA from the chloroeremomycin biosynthetic pathway. Proc Natl Acad Sci U S A 2003, 100:9238-9243.

46. Mulichak AM, Lu W, Losey HC, Walsh CT, Garavito RM: Crysta structure of vancosaminyltransferase GtfD from the vancomycin biosynthetic pathway: interactions with acceptor and nucleotide ligands. Biochemistry 2004, 43:5।70-5 I80.

47. Shao H, He X, Achnine L, Blount JW, Dixon RA, Wang X: Crysta structures of a multifunctional triterpene/flavonoid glycosyl- transferase from Medicago truncatula. Plant Cell 2005, |7:3|4|-3|54

48. Rossmann MG, Moras D, Olsen KW: Chemical and biological evolution of nucleotide-binding protein. Nature 1974, 250:194-199.

49. Radominska-Pandya A, Czernik PJ, Little JM, Battaglia E, Mackenzie PI: Structural and functional studies of UDP-glucuronosyltransferases. Drug Metab Rev 1999, 3 I:817-899.

50. Labrune P, Myara A, Hadchouel M, Ronchi F, Bernard O, Trivin F Chowdhury NR, Chowdhury JR, Munnich A, Odievre M: Genetic heterogeneity of Crigler-Najjar syndrome type I: a study of I4 cases. Hum Genet 1994, 94:693-697.

5I. Ciotti M, Chen F, Rubaltelli FF, Owens IS: Coding defect and a TATA box mutation at the bilirubin UDP-glucuronosyltransferase gene cause Crigler-Najjar type I disease. Biochim Biophys Acta 1998, I 407:40-50.

52. Servedio V, d'Apolito M, Maiorano N, Minuti B, Torricelli F, Ronchi F, Zancan L, Perrotta S, Vajro P, Boschetto L, lolascon A: Spectrum of UGTIAI mutations in Crigler-Najjar (CN) syndrome patients: identification of twelve novel alleles and genotypephenotype correlation. Hum Mutat 2005, 25:325.

53. D'Apolito M, Marrone A, Servedio V, Vajro P, De Falco L, Iolascon A Seven novel mutations of the UGTIAI gene in patients with unconjugated hyperbilirubinemia. Haematologica 2007 92:133-134

54. Erps LT, Ritter JK, Hersh JH, Blossom D, Martin NC, Owens IS: Identification of two single base substitutions in the UGTI gene locus which abolish bilirubin uridine diphosphate glucuronosyltransferase activity in vitro. J Clin Invest 1994, 93:564-570.

55. Ciotti M, Obaray R, Martin MG, Owens IS: Genetic defects at the UGTI locus associated with Crigler-Najjar type I disease, including a prenatal diagnosis. Am J Med Genet 1997, 68:173-178.

56. Ciotti M, Werlin SL, Owens IS: Delayed response to phenobarbital treatment of a Crigler-Najjar type II patient with partially inactivating missense mutations in the bilirubin UDP-glucuronosyltransferase gene. I Pediatr Gastroenterol Nutr 1999, 28:210-213.

57. Moghrabi N, Clarke DJ, Boxer M, Burchell B: Identification of an Ato-G missense mutation in exon 2 of the UGT I gene complex that causes Crigler-Najiar syndrome type 2. Genomics 1993, 18:17|-173.

58. Labrune P, Myara A, Chalas J, Le Bihan B, Capel L, Francoual J: Association of a homozygous (TA) 8 promoter polymorphism and a N400D mutation of UGTIAI in a child with Crigler-Najjar type II syndrome. Hum Mutat 2002, 20:399-40I.

59. Takeuchi K, Kobayashi Y, Tamaki S, Ishihara T, Maruo Y, Araki J, Mifuji R, Itani T, Kuroda M, Sato H, Kaito M, Adachi Y: Genetic polymorphisms of bilirubin uridine diphosphate-glucuronosyltransferase gene in Japanese patients with Crigler-Najjar syndrome or Gilbert's syndrome as well as in healthy Japanese subjects. I Gastroenterol Hepatol 2004, 19: 1023-1028.

60. Koiwai O, Nishizawa M, Hasada K, Aono S, Adachi Y, Mamiya N, Sato $\mathrm{H}$ : Gilbert's syndrome is caused by a heterozygous missense mutation in the gene for bilirubin UDP-glucuronosyltransferase. Hum Mol Genet 1995, 4:1 I83-II86.

61. Aono S, Adachi Y, Uyama E, Yamada Y, Keino H, Nanno T, Koiwai O, Sato H: Analysis of genes for bilirubin UDP-glucuronosyltransferase in Gilbert's syndrome. Lancet 1995, 345:958-959.

62. Bonnett $R$, Davies JE, Hursthouse MB: Structure of bilirubin. Nature 1976, 262:326-328.

63. Jones G, Willett P, Glen RC, Leach AR, Taylor R: Development and validation of a genetic algorithm for flexible docking. I Mol Biol 1997, 267:727-748.

64. Petit F, Gajdos V, Capel L, Parisot F, Myara A, Francoual J, Labrune P: Crigler-Najiar type II syndrome may result from several types and combinations of mutations in the UGTIAI gene. Clin Genet 2006, 69:525-527.

65. Sutomo R, Laosombat V, Sadewa AH, Yokoyama N, Nakamura H, Matsuo $M$, Nishio $H$. Novel missense mutation of the UGTIAI gene in Thai siblings with Gilbert's syndrome. Pediatr Int 2002, 44:427-432.

66. Ghosh SS, Lu Y, Lee SW, Wang X, Guha C, Roy-Chowdhury J, RoyChowdhury $\mathrm{N}$ : Role of cysteine residues in the function of human UDP glucuronosyltransferase isoform IAI (UGTIAI). Biochem / 2005, 392:685-692.

67. Seppen J, Bosma PJ, Goldhoorn BG, Bakker CT, Chowdhury JR, Chowdhury NR, Jansen PL, Oude Elferink RP: Discrimination between Crigler-Najjar type I and II by expression of mutant bilirubin uridine diphosphate-glucuronosyltransferase. J Clin Invest 1994 94:2385-2391.

68. Bosma PJ, Goldhoorn B, Oude Elferink RP, Sinaasappel M, Oostra BA Jansen PL: A mutation in bilirubin uridine 5 '-diphosphate-glu- 
curonosyltransferase isoform I causing Crigler-Najiar syndrome type II. Gastroenterology 1993, 105:216-220.

69. Iolascon A, Meloni A, Coppola B, Rosatelli MC: Crigler-Najjar syndrome type II resulting from three different mutations in the bilirubin uridine 5'-diphosphate-glucuronosyltransferase (UGTIAI) gene. J Med Genet 2000, 37:7। 2-7/3.

70. Su C, Jakobsen I, Gu X, Nei M: Diversity and evolution of T-cell receptor variable region genes in mammals and birds. Immunogenetics 1999, 50:301-308.

71. Emes RD, Beatson SA, Ponting CP, Goodstadt L: Evolution and comparative genomics of odorant- and pheromone-associated genes in rodents. Genome Res 2004, 14:591-602.

72. Yang Z: PAML: a program package for phylogenetic analysis by maximum likelihood. Comput Appl Biosci 1997, 13:555-556.

73. Lawrence CE, Altschul SF, Boguski MS, Liu JS, Neuwald AF, Wootton JC: Detecting subtle sequence signals: a Gibbs sampling strategy for multiple alignment. Science 1993, 262:208-214 [http:// bayesweb.wadsworth.org/gibbs/gibbs.html].

74. Crooks GE, Hon G, Chandonia JM, Brenner SE: WebLogo: a sequence logo generator. Genome Res 2004, I 4: I | 88- I I 90 [http:/ /weblogo.berkeley.edu/logo.cgi].

75. Jones DT: Protein secondary structure prediction based on position-specific scoring matrices. J Mol Biol 1999, 292:195-202.

76. Kneller DG, Cohen FE, Langridge R: Improvements in protein secondary structure prediction by an enhanced neural network. J Mol Biol 1990, 214:171-182.

77. Krogh A, Brown M, Mian IS, Sjolander K, Haussler D: Hidden Markov models in computational biology. Applications to protein modeling. J Mol Biol 1994, 235:150|-1531 [http:// www.cse.ucsc.edu/research/compbio/sam.html]

78. Guex N, Peitsch MC: SWISS-MODEL and the Swiss-PdbViewer: an environment for comparative protein modeling. Electrophoresis 1997, 18:27|4-2723.

79. Melo F, Feytmans E: Assessing protein structures with a nonlocal atomic interaction energy. I Mol Biol 1998, 277: | |4|-II52

80. Eisenberg $D$, Luthy R, Bowie JU: VERIFY3D: assessment of protein models with three-dimensional profiles. Methods Enzymol 1997, 277:396-404.

81. Laskowski RA, W. MAM, Moss DS, Thornton JM: PROCHECK: program to check the stereochemical quality of protein structures. J Appl Cryst 1993, 26:283-291.

82. Nicholas KB, Nicholas HBJ, Deerfield DWII: GeneDoc: Analysis and Visualization of Genetic Variation. EMBNEWNEWS 1997, 4:14 [http://www.nrbsc.org/gfx/genedoc/index.html].

83. DeLano WL: The PyMOL Molecular Graphics System. (2002) DeLano Scientific, Palo Alto, CA, USA. 2002 [http:// www.pymol.org].
Publish with Bio Med Central and every scientist can read your work free of charge

"BioMed Central will be the most significant development for disseminating the results of biomedical research in our lifetime. "

Sir Paul Nurse, Cancer Research UK

Your research papers will be:

- available free of charge to the entire biomedical community

- peer reviewed and published immediately upon acceptance

- cited in PubMed and archived on PubMed Central

- yours - you keep the copyright
BioMedcentral 